TRANSACTIONS OF THE

AMERICAN MATHEMATICAL SOCIETY

Volume 357, Number 7, Pages 2681-2722

S 0002-9947(04)03524-X

Article electronically published on April 16, 2004

\title{
GOOD MEASURES ON CANTOR SPACE
}

\author{
ETHAN AKIN
}

\begin{abstract}
While there is, up to homeomorphism, only one Cantor space, i.e. one zero-dimensional, perfect, compact, nonempty metric space, there are many measures on Cantor space which are not topologically equivalent. The clopen values set for a full, nonatomic measure $\mu$ is the countable dense subset $\{\mu(U): U$ is clopen $\}$ of the unit interval. It is a topological invariant for the measure. For the class of good measures it is a complete invariant. A full, nonatomic measure $\mu$ is good if whenever $U, V$ are clopen sets with $\mu(U)<$ $\mu(V)$, there exists $W$ a clopen subset of $V$ such that $\mu(W)=\mu(U)$. These measures have interesting dynamical properties. They are exactly the measures which arise from uniquely ergodic minimal systems on Cantor space. For some of them there is a unique generic measure-preserving homeomorphism. That is, within the Polish group of such homeomorphisms there is a dense, $G_{\delta}$ conjugacy class.
\end{abstract}

\section{INTRODUCTION}

A Cantor space is a nonempty, compact, perfect, zero-dimensional metric space. An ordered Cantor space is a Cantor space equipped with a closed total order so that the order topology agrees with the metric topology. Any two Cantor spaces are homeomorphic and any two ordered Cantor spaces are order isomorphic.

A Borel probability measure $\mu$ on a compact metric space $X$ is called full when nonempty open subsets have positive measure and nonatomic when countable subsets have measure zero. There exist many homeomorphically distinct, full, nonatomic measures on Cantor space. In fact the clopen values set $S(\mu)$, defined to be the set of values of the measure on the clopen subsets of $X$, provides a topological invariant, although it is not a complete invariant. On the other hand, if $X$ is ordered, then the special clopen values set $\tilde{S}(\mu)$ is defined to be the set of values of the measure on those clopen intervals in $X$ which contain the minimum point $m$ of $X . \tilde{S}(\mu)$ is a complete invariant with respect to a measure preserving, order isomorphism. These results are proved in Akin 1999 and are reviewed in Section 1 below. The same set, which they called the gap invariant, is introduced by Cooper and Pignataro [1988] for a related purpose.

A full, nonatomic measure $\mu$ on a Cantor space $X$ is said to satisfy the Subset Condition if for all clopen subsets $U$ and $V$ of $X, \mu(U) \leq \mu(V)$ implies that there exists a clopen subset $U_{1}$ of $V$ such that $\mu(U)=\mu\left(U_{1}\right)$. $\mu$ is called good when it

Received by the editors April 9, 2002 and, in revised form, July 24, 2003.

2000 Mathematics Subject Classification. Primary 37A05, 28D05; Secondary 37B10, 54H20.

Key words and phrases. Cantor set, measure on Cantor space, ordered measure spaces, unique ergodicity, generic conjugacy class, Rohlin property. 
satisfies the Subset Condition. On the other hand, a measure $\mu$ and an order $\leq$ are adapted if $S(\mu)=\tilde{S}(\mu)$.

Theorem 0.1. If a measure $\mu$ is adapted to the order of an ordered Cantor space $X$, then it is a good measure on $X$. Conversely, if $\mu$ is a good measure on a Cantor space $X$, then there exists an order on $X$ which is adapted to $\mu$.

Together with its applications, this is the main result of Section 2. From this theorem it follows that the clopen values set is a complete invariant for good measures. If $\mu$ is good on $X$, then for any nonempty clopen subset $V$ the relative measure $\mu_{V}$ is good on $V$. It then follows that if $U$ and $V$ are clopen subsets with the same measure, then there exists a measure preserving automorphism of $X$ mapping $U$ to $V$. From a sharpening of the theorem one can prove that if $\mu$ is good, then the group of $\mu$ preserving automorphisms acts transitively on $X$.

A subset $S$ of the unit interval $I$ which contains 0,1 is called group-like if $S=$ $G \cap I$ with $G$ an additive subgroup of $\mathbb{R}$, or, equivalently, if $S+\mathbb{Z}$ is a group. If $S+\mathbb{Z}$ is a rational vector space (or a subfield of $\mathbb{R}$ ), then we call $S \mathbb{Q}$-like (resp. field-like).

If $\mu$ is good, then $S(\mu)$ is group-like and $\mu$ is adapted to an order iff $\tilde{S}(\mu)$ is grouplike. The other conditions are associated with stronger homogeneity conditions on $\mu$. In Section 3 we prove that goodness of $\mu$ with $S(\mu)$ field-like is equivalent to the condition that for every nonempty clopen subset $V$ of $X$ the relative measure $\mu_{V}$ is homeomorphic to $\mu$ on $X$, together with the technical condition that $\alpha \in S(\mu)$ with $\alpha<1 / 2$ implies $2 \alpha \in S(\mu)$. I suspect that this conditon, a weakening of the assumption that $S(\mu)$ is group-like, is redundant, but I have been unable to eliminate it.

In Section 4 we consider automorphisms of good measures. Assume $\mu$ is a good measure on $X$. If $S(\mu) \cap \mathbb{Q}$ is infinite, then there is a $\mu$ preserving automorphism which is uniquely ergodic and which has an adding machine translation as an almost one-to-one factor. On the other hand, if $\alpha$ is an irrational in $S(\mu)$, then there is a $\mu$ preserving automorphism which is uniquely ergodic and which has translation by $\alpha$ on the circle $\mathbb{R} / \mathbb{Z}$ as an almost one-to-one factor. One of these two cases always applies and so every good measure is the invariant measure for some uniquely ergodic transformation on Cantor space. Glasner and Weiss [1995] prove the converse, that is, the invariant measure for a uniquely ergodic transformation on Cantor space is good. Together these results relate this little theory to the powerful machinery due to Giordano, Putnam and Skau 1995. Indeed, in his review of recent results in topological dynamics 2002, Glasner shows how to derive several of our results from theirs, in particular avoiding our use of the order structure. On the other hand, the order structure allows us to make very explicit constructions.

The final result of Section 4 concerns the Rohlin Property. A Polish topological group $G$ is said to satisfy the Rohlin Property if the adjoint action of $G$ on $G$ is topologically transitive, i.e. $\operatorname{TRANS}(G)=_{\operatorname{def}}\{x \in G: \operatorname{ad}(G) x$ is dense in $G\}$ is nonempty, in which case, $\operatorname{TRANS}(G)$ is a dense $G_{\delta}$ set. A compact metric space $X$ is said to satisfy the Rohlin Property when its automorphism group does. In Glasner and Weiss [2001] and Akin, Hurley and Kennedy [2003] it is proved that Cantor space satisfies the Rohlin Property. Under some circumstances the group of $\mu$ preserving homeomorphisms satisfies a more powerful condition which we call the Strong Rohlin Property: 
Theorem 0.2. If $\mu$ is a good measure on $X$ with $S(\mu) \mathbb{Q}$-like, then there exists a single, necessarily unique, conjugacy class which is a dense $G_{\delta}$ subset of the group of measure preserving homeomorphisms on $X$.

In such a case, we can speak of the generic homeomorphism on $X$. We show that it is conjugate to the product of the identity on $X$ with the translation map of the universal adding machine.

\section{The ClOPEn VALUES INVARIANTS}

All of our topological spaces will be nonempty Polish spaces. That is, they are separable and completely metrizable. Furthermore, unless we mention otherwise, our spaces $X, Y$ are assumed to be compact as well. Such a space is a Cantor space when it is zero-dimensional and perfect. One kind of example is a Cantor set, a closed, perfect, nowhere dense subset of the unit interval $I=[0,1]$ in $\mathbb{R}$. If $F$ is a discrete finite space containing at least two points and $\mathbb{Z}_{+}=\{0,1,2, \ldots\}$, then the product space $F^{\mathbb{Z}_{+}}$is a Cantor space as well.

An order $\leq$ on a space $X$ is a total order, i.e. any two points are comparable, which is closed as a subset of $X \times X$, or, equivalently, whose order topology agrees with the original compact topology on $X$. An ordered (Cantor) space is a pair $(X, \leq)$ where $X$ is a (Cantor) space and $\leq$ is an order on $X$. Thus, $(X, \leq)$ is a compact linearly ordered topological space (acronym: LOTS). Such a space has a maximum element and a minimum element which we will denote by $M$ and $m$, respectively. We will adopt the usual interval notation so that, for example, $[x, y)=\{z \in X: x \leq z<y\}$.

A point $x$ is called a left endpoint if the interval $[m, x]$ is clopen and a right endpoint if $[x, M]$ is clopen. Thus, $M$ is a left endpoint and $m$ is a right endpoint. A point is both a left and right endpoint iff it is an isolated point.

If $X$ is perfect, i.e. it has no isolated points, then the endpoints other than $m, M$ occur in pairs. If $\left[m, x_{-}\right]$is clopen with $x_{-} \neq M$, then

$$
X \backslash\left[m, x_{-}\right]=\left[x_{+}, M\right] \quad \text { with } \quad x_{+}=\min \left(X \backslash\left[m, x_{-}\right]\right) .
$$

Such an endpoint pair $\left\{x_{-}, x_{+}\right\}$is characterized by the conditions that $x_{-}<x_{+}$ but the open interval $\left(x_{-}, x_{+}\right)$is empty.

An order map $f:\left(X_{1}, \leq\right) \rightarrow\left(X_{2}, \leq\right)$ is a continuous map of spaces such that for $x, y \in X_{1}$

$$
x \leq y \quad \Longrightarrow \quad f(x) \leq f(y) .
$$

If $[a, b]$ is a closed subinterval of $X_{2}$, then

$$
\begin{aligned}
f^{-1}([a, b]) & =[x, y] \quad \text { with } \\
x=\operatorname{minf}^{-1}([a, b]) \quad \text { and } y & =\operatorname{maxf}^{-1}([a, b]) .
\end{aligned}
$$

If an order map $f$ is a homeomorphism, then $f^{-1}$ is an order map as well and we call $f$ an order isomorphism.

Any two Cantor spaces are homeomorphic and any two ordered Cantor spaces are order isomorphic. We refer to these results as The Uniqueness of Cantor (see Akin [1999] Proposition 1.2 and Corollary 2.13 for examples of proofs of these classic results). In particular, any ordered Cantor space is order isomorphic to the classical Cantor set in $I$. The left and right endpoints of an endpoint pair correspond to the left and right endpoints of one of the open intervals in $I$ complementary to the 
Cantor set. Hence, the sets of left and right endpoints are each countable dense subsets of any ordered Cantor space.

A continuous map $f: X_{1} \rightarrow X_{2}$ is called almost open if for $A \subset X_{1}$

$$
\operatorname{Int} A \neq \emptyset \quad \Longrightarrow \quad \operatorname{Intf}(A) \neq \emptyset .
$$

The map is called almost one-to-one if $I n j_{f}$ is dense in $X_{1}$ where

$$
\operatorname{Inj}_{f} \quad=_{\text {def }} \quad\left\{x \in X_{1}: f^{-1}(f(x))=\{x\}\right\} .
$$

Lemma 1.1. Let $f:\left(X_{1}, \leq\right) \rightarrow\left(X_{2}, \leq\right)$ be a surjective order preserving map with closed point images, i.e. $f^{-1}(y)$ is closed in $X_{1}$ for all $y \in X_{2}$.

(a) $f$ is continuous and so is an order map.

(b) $f\left(m_{1}\right)=m_{2}$ and $f\left(M_{1}\right)=M_{2}$.

(c) If $y_{-}, y_{+}$is an endpoint pair in $X_{2}$, then there exists an endpoint pair $x_{-}, x_{+}$in $X_{1}$ such that

$$
f\left(x_{ \pm}\right)=y_{ \pm} .
$$

(d) If $x_{-}, x_{+}$is an endpoint pair in $X_{1}$, then either $f\left(x_{-}\right)=f\left(x_{+}\right)$or $f\left(x_{-}\right)$, $f\left(x_{+}\right)$is an endpoint pair in $X_{2}$.

(e) If $X_{1}$ and $X_{2}$ are perfect, then the following conditions are equivalent:

(1) $X_{1} \backslash$ Inj $_{f}$ is countable.

(2) $f$ is almost one-to-one.

(3) $f$ is almost open.

(4) For all $z \in X_{2}, f^{-1}(z)$ is nowhere dense.

(5) For all $z \in X_{2}, f^{-1}(z)$ is finite.

(6) For all $x, y \in X_{1}, f(x)=f(y)$ implies either $x=y$ or $x, y$ is an endpoint pair.

Proof. (a) Observe first that since $f$ is surjective we can sharpen (1.3):

$$
\begin{array}{r}
f^{-1}([a, b])=[x, y] \quad \text { with } \\
x=\operatorname{minf}^{-1}(a) \quad \text { and } y=\operatorname{maxf}^{-1}(b),
\end{array}
$$

and similarly,

$$
\begin{array}{r}
f^{-1}((a, b)) \quad=(\tilde{x}, \tilde{y}) \quad \text { with } \\
\tilde{x}=\operatorname{maxf}^{-1}(a) \quad \text { and } \quad \tilde{y}=\operatorname{minf}^{-1}(b) .
\end{array}
$$

It follows that $f$ is continuous and so is an order map.

(b) If $A \subset X_{1}$ is closed, then

$$
f(\max A)=\max f(A) \quad \text { and } \quad f(\min A)=\operatorname{minf}(A) .
$$

Since $f$ is surjective, (b) follows.

(c) From (1.8) this follows with

$$
x_{-}=\operatorname{maxf}^{-1}\left(y_{-}\right) \quad \text { and } \quad x_{+}=\operatorname{minf}^{-1}\left(y_{+}\right) .
$$

(d) If the interval $(f(x), f(y))$ is nonempty, then there exists $z \in X_{1}$ with $f(x)<$ $f(z)<f(y)$. Because $f$ is an order map, $x<z<y$ and so $x, y$ is not an endpoint pair.

(e) (4) $\Rightarrow(6)$ : Assume $x<y$ with $z=f(x)=f(y)$ but $x, y$ is not an endpoint pair. Since $f$ is an order map, $f^{-1}(z)$ contains the nonempty open interval $(x, y)$. Since $X_{2}$ is perfect, $\operatorname{Int}\{z\}=\emptyset$. Hence, $f$ is not almost open. 
(6) $\Rightarrow(5)$ : Obvious.

$(5) \Rightarrow(4)$ : Obvious since $X_{1}$ is perfect.

$(6) \Rightarrow(1): X_{1} \backslash I n j_{f}$ is a subset of the countable set of endpoints.

$(1) \Rightarrow(2)$ : Since $X_{1}$ is perfect and compact, the Baire Category Theorem implies that the complement of a countable set is dense.

$(2) \Rightarrow(3)$ : Any continuous, almost one-to-one map is almost open. In fact, from compactness it is easy to check that if $U \subset X_{1}$ is open, then

$$
f\left(U \cap \operatorname{Inj}_{f}\right) \subset \operatorname{Intf}(U) .
$$

$(3) \Rightarrow(4)$ : Because $X_{2}$ is perfect, (1.4) implies that $f^{-1}(z)$ is nowhere dense.

Remark. The unit interval $I$ has no endpoint pairs. Hence, for $f:(X, \leq) \rightarrow(I, \leq)$ an almost one-to-one surjection with $X$ perfect, if $x<y$ in $X$, then

$$
f(x)=f(y) \quad \Longleftrightarrow \quad\{x, y\} \text { is an endpoint pair in } X .
$$

Following Akin [1999] we study measures on Cantor spaces. A measure $\mu$ on a space $X$ is a Borel probability measure, i.e. $\mu(X)=1$. The measure is full if nonempty open sets have positive measure and nonatomic if countable sets have zero measure. Let $\mathcal{M}_{X}$ denote the set of full, nonatomic measures on $X$. For example, the Lebesgue measure $\lambda$ is an element of $\mathcal{M}_{I}$. Notice that the existence of a full, nonatomic measure implies that the space $X$ is perfect.

When we speak of a metric $d$ on any metrizable topological space $Z$ we mean a metric whose associated topology is original topology on $Z$. If $A \subset Z$ we define the diameter of $A$ by

$$
d(A)=\sup \{d(x, y): x, y \in A\},
$$

with $d(\emptyset)=0$ by convention.

Lemma 1.2. Let $d$ be a metric on a space $X$ and let $\mu$ be a nonatomic measure on $X$. For every $\epsilon>0$ there exists a $\delta>0$ such that for every Borel set $A \subset X$

$$
d(A)<\delta \quad \Longrightarrow \quad \mu(A)<\epsilon .
$$

Proof. Since $\mu$ is nonatomic, every point $x \in X$ is contained in some open set $U_{x}$ with $\mu\left(U_{x}\right)<\epsilon$. By compactness, the open cover $\left\{U_{x}: x \in X\right\}$ has a positive Lebesgue number $\delta$. That is, $d(A)<\delta$ implies $A \subset U_{x}$ for some $x$.

If $(X, \leq)$ is an ordered space and $\mu \in \mathcal{M}_{X}$, then the cumulative distribution function, hereafter the CDF, of $\mu$ is the order map $F_{\mu}:(X, \leq) \rightarrow(I, \leq)$ defined by

$$
F_{\mu}(x) \quad=_{\text {def }} \quad \mu([m, x]) .
$$

Since $\mu$ is nonatomic we have for $x<y$ in $X$

$$
\mu((x, y))=\mu([x, y])=F_{\mu}(y)-F_{\mu}(x) .
$$

In particular, if $x_{-}, x_{+}$is an endpoint pair in $X$, then

$$
F_{\mu}\left(x_{-}\right)=F_{\mu}\left(x_{+}\right) .
$$


Proposition 1.3. If $(X, \leq)$ is a perfect, ordered space, then the association $\mu \mapsto F_{\mu}$ defines a bijection from the set $\mathcal{M}_{X}$ of full, nonatomic measures on $X$ to the set of almost one-to-one surjections of $(X, \leq)$ onto $(I, \leq)$.

Proof. When the measure $\mu$ is nonatomic, it is easy to check that the CDF is continuous and surjective. Because the measure is full, the CDF is almost open and so, by Lemma 1.1(e) it is almost one-to-one. Begining with a continuous, almost one-to-one surjection one defines the measure on intervals via (1.16) and, then applies the usual Lebesgue-Stieltjes construction. See Akin [1999] Propositions 2.5 and 2.6 .

If $f: X_{1} \rightarrow X_{2}$ is a continuous map and $\mu$ is a measure on $X_{1}$, then the induced measure $f_{*} \mu$ on $X_{2}$ is defined by

$$
f_{*} \mu(B) \quad=_{\text {def }} \quad \mu\left(f^{-1}(B)\right)
$$

for every Borel subset $B$ of $X_{2}$.

Assume $\mu \in \mathcal{M}_{X_{1}}$. If $f$ is surjective, then $f_{*} \mu$ is full and if $f^{-1}(z)$ is countable for every $z \in X_{2}$, then $f_{*} \mu$ is nonatomic. If both these conditions on $f$ hold, then $f_{*} \mu \in \mathcal{M}_{X_{2}}$. In particular, any homeomorphism induces a bijection from $\mathcal{M}_{X_{1}}$ to $\mathcal{M}_{X_{2}}$. If $(X, \leq)$ is a perfect ordered space and $\mu \in \mathcal{M}_{X}$, then by Akin [1999] Corollary 2.8, the CDF $F_{\mu}$ maps $\mu$ to Lebesgue measure $\lambda$ on $I$. That is,

$$
F_{\mu *} \mu=\lambda \text {. }
$$

Following Akin 1999] we define invariants by using the clopen subsets. For a space $X$ and $\mu \in \mathcal{M}_{X}$ define the clopen values set for $\mu$

$$
S(\mu) \quad=_{\text {def }} \quad\{\mu(U): U \text { is clopen in } X\} .
$$

If $(X, \leq)$ is an ordered space, then the special clopen values set for $\mu$ is

$$
\tilde{S}(\mu) \quad=_{\text {def }} \quad\left\{F_{\mu}(x): x \text { is an endpoint of } X\right\} .
$$

For any $A \subset \mathbb{R}$ let $G[A]$ denote the additive group generated by $A$, so that $G[A]$ is the set of all finite sums of differences of elements of $A$ (including $0=$ the empty sum so that $G[\emptyset]=\{0\})$.

Proposition 1.4. $\quad$ (a) Let $X$ be a space and $\mu \in \mathcal{M}_{X} . S(\mu)$ is a countable subset of I containing 0,1 . If $X$ is a Cantor space, then $S(\mu)$ is dense in I.

(b) Let $(X, \leq)$ be an ordered space and $\mu \in \mathcal{M}_{X} . \tilde{S}(\mu)$ is a countable subset of $I$ containing 0,1 . If $X$ is a Cantor space, then $\tilde{S}(\mu)$ is dense in $I$. Furthermore,

$$
\tilde{S}(\mu) \quad \subset \quad S(\mu) \quad \subset \quad G[\tilde{S}(\mu)] \cap I .
$$

Proof. (a) Since $X$ has a countable base and any clopen subset is a finite union of elements of the base, there are only countably many clopen subsets of $X$, including $\emptyset$ and $X$. If $X$ is a Cantor space, then we can choose an order, by Uniqueness of Cantor, and then apply (b) to obtain density of $S(\mu)$.

(b) $F_{\mu}(m)=0, F_{\mu}(M)=1$ and for any left endpoint $x, F_{\mu}(x)=\mu(U)$, where $U=[m, x]$ is clopen. Hence $\tilde{S}(\mu) \subset S(\mu)$ and so is countable by (a). Every clopen set is a finite disjoint union of clopen intervals and so by $(1.16) S(\mu) \subset G[\tilde{S}(\mu)]$. If $X$ is a Cantor space, then the set of endpoints is dense in $X$ and so its image $\tilde{S}(\mu)$ under the surjection $F_{\mu}$ is dense in $I$. 
Proposition 1.5. For $\alpha=1,2$ let $X_{\alpha}$ be a space and $\mu_{\alpha} \in \mathcal{M}_{X_{\alpha}}$. Let $f: X_{1} \rightarrow X_{2}$ be a continuous map. If $f_{*} \mu_{1}=\mu_{2}$, then $f$ is a surjection and

$$
S\left(\mu_{2}\right) \quad \subset \quad S\left(\mu_{1}\right)
$$

with equality if $f$ is a homeomorphism.

Proof. The preimage of $X_{2} \backslash f\left(X_{1}\right)$ is empty and so $X_{2} \backslash f\left(X_{1}\right)$ is an open set with $\mu_{2}$ measure zero. Since $\mu_{2}$ is full, $f$ is surjective. Since the preimage of a clopen set is clopen, (1.23) follows. If $f$ is a homeomorphism, then we apply (1.23) to $f^{-1}$ to get equality.

Thus, $S(\mu)$ is a homeomorphism invariant for the measure $\mu$. It is not a complete invariant in general. On the other hand, $\tilde{S}(\mu)$ is a complete order isomorphism invariant. For completeness we will sketch the proof of this and of some other, related, results from Akin [1999.

Theorem 1.6. For $\alpha=1,2$ let $\left(X_{\alpha}, \leq\right)$ be a space and $\mu_{\alpha} \in \mathcal{M}_{X_{\alpha}}$.

(a) For a continuous map $f: X_{1} \rightarrow X_{2}$ the following conditions are equivalent:

(1) $f$ is an order map and $f_{*} \mu_{1}=\mu_{2}$.

(2) $F_{\mu_{1}}=F_{\mu_{2}} \circ f$.

If these conditions hold, then $f$ is an almost one-to-one surjection and

$$
\tilde{S}\left(\mu_{2}\right) \quad \subset \quad \tilde{S}\left(\mu_{1}\right)
$$

with equality if $f$ is a homeomorphism.

(b) If $\tilde{S}\left(\mu_{2}\right) \subset \tilde{S}\left(\mu_{1}\right)$, then there is a unique continuous map $f: X_{1} \rightarrow X_{2}$ such that $F_{\mu_{1}}=F_{\mu_{2}} \circ$ f. If $\tilde{S}\left(\mu_{2}\right)=\tilde{S}\left(\mu_{1}\right)$, then this map is a homeomorphism.

Proof. The closed subset $F=\left(F_{\mu_{2}}\right)^{-1} \circ F_{\mu_{1}} \subset X_{1} \times X_{2}$ can be regarded as a relation from $X_{1}$ to $X_{2}$. It is a surjective relation, that is, $F(x) \neq \emptyset$ for all $x \in X_{1}$ and $F^{-1}(y) \neq \emptyset$ for all $y \in X_{2}$. Furthermore, on the complement of the countable set

$$
S^{*} \quad=_{\text {def }}\left(F_{\mu_{1}}\right)^{-1}\left(\tilde{S}\left(\mu_{2}\right) \cup \tilde{S}\left(\mu_{1}\right)\right)
$$

the relation $F$ resticts to a well-defined, injective function. In fact, if $x<y$ in $X_{1} \backslash S^{*}$, then

$$
F_{\mu_{2}}(F(x))=F_{\mu_{1}}(x)<F_{\mu_{1}}(y)=F_{\mu_{2}}(F(y)),
$$

and so $F(x)<F(y)$. Since $X_{1}$ and $X_{2}$ are perfect, $X_{1} \backslash S^{*}$ is dense in $X_{1}$ and its image, which also has a countable complement, is dense in $X_{2}$.

(a), (2) $\Rightarrow(1)$ : Condition (2) implies that the relation $F$ is equal to $f$ on $X_{1} \backslash S^{*}$. Because it is continuous and is order preserving on the dense set $X_{1} \backslash S^{*}, f$ is an order map. Because it has a dense image, $f$ is surjective. Since $X_{1} \backslash S^{*} \subset I n j_{f}$, $f$ is almost one-to-one. Furthermore, there is at most one continuous map which satisfies (2). This proves uniqueness in (b). Furthermore, condition (2) implies that $\mu_{2}$ and $f_{*} \mu_{1}$ agree on all intervals of the form $[m, x]$ and so by (1.16) on all intervals. Since the intervals generate the Borel sets, $f_{*} \mu_{1}=\mu_{2}$. Finally, Lemma 1.1(c) and (2) imply (1.24).

$(1) \Rightarrow(2)$ : This is an easy exercise. See, e.g., Akin [1999] Lemma 2.7. As before when $f$ is a homeomorphism we obtain equality by applying the result to $f^{-1}$.

(b) Since uniqueness has already been established in the proof of part (a), the remainder is the Lifting Lemma 2.9 of Akin [1999]. The key step is the extension of $f=F$ on $X_{1} \backslash S^{*}$. Each endpoint pair of $X_{1}$ is in $S^{*}$ and is associated by $F$ either 
to single point or an endpoint pair in $X_{2}$. By assumption (1.24) each endpoint pair in $X_{2}$ comes from an endpoint pair in $X_{1}$. The extension maps the left endpoint in $X_{1}$ to the left endpoint in $X_{2}$ and the right to the right.

Let $\mathcal{D}$ denote the uncountable set of countable, dense subsets of the unit interval $I$ which contain 0,1 . Let $\mathcal{G}$ denote the automorphism group of $(I, \leq)$, that is, the group of order isomorphisms from $(I, \leq)$ to itself. So $\phi \in \mathcal{G}$ when $\phi$ is an increasing real-valued funtion on $I$ which fixes 0 and 1. By Lemma 1.1(d), if $\phi$ is an almost one-to-one, surjective order map on $(I, \leq)$, then $\phi \in \mathcal{G}$.

Theorem 1.7. Let $(X, \leq)$ be an ordered Cantor space.

(a) The group $\mathcal{G}$ acts on the set $\mathcal{M}_{X}$. For $\left(\phi, \mu_{1}\right) \in \mathcal{G} \times \mathcal{M}_{X}$ and $\mu_{2} \in \mathcal{M}_{X}$

$$
\mu_{2}=\phi \mu_{1} \quad \Longleftrightarrow \quad F_{\mu_{2}}=\phi \circ F_{\mu_{1}} .
$$

The action is transitive and free, i.e. for $\mu_{1} \in \mathcal{M}_{X}$ the map $\phi \mapsto \phi \mu_{1}$ is a bijection from $\mathcal{G}$ to $\mathcal{M}_{X}$.

(b) The group $\mathcal{G}$ acts on the set $\mathcal{D}$. For $\left(\phi, D_{1}\right) \in \mathcal{G} \times \mathcal{D}$

$$
D_{2}=\phi D_{1} \quad \Longleftrightarrow \quad D_{2}=\phi\left(D_{1}\right) .
$$

The action is transitive, i.e. for $D_{1} \in \mathcal{D}$ the map $\phi \mapsto \phi D_{1}$ is a surjection from $\mathcal{G}$ to $\mathcal{D}$.

(c) The map $\tilde{S}: \mathcal{M}_{X} \rightarrow \mathcal{D}$ is $\mathcal{G}$ equivariant, i.e. for $(\phi, \mu) \in \mathcal{G} \times \mathcal{M}_{X}$

$$
\tilde{S}(\phi \mu)=\phi \tilde{S}(\mu) .
$$

$\tilde{S}$ induces a bijection between the order isomorphism classes in $\mathcal{M}_{X}$ and the elements of $\mathcal{D}$.

Proof. This result is Theorem 3.2 together with Corollary 3.3 of Akin [1999. We sketch the proof of some of the results. The action in (a) is well defined by Proposition 1.3. Transitivity of the action in (b) follows from the classical result that any countable dense subset of $I$ which contians 0 and 1 is order isomorphic to the set of rationals in $I$. The map $\tilde{S}$ in (c) is obviously equivariant. Since Theorem 1.6 implies that $\tilde{S}(\mu)$ provides a complete order isomorphism invariant for $\mu$, it follows that each $(\tilde{S})^{-1}(D)$ is an order isomorphism class.

For a space $X$ let $H(X)$ denote the automorphism group of the space, i.e. the group of homeomorphisms on $X$. Equipped with the topology of uniform convergence, $H(X)$ is a Polish group, that is, a completely metrizable, separable topological group. If $\mu$ is a measure on $X$, then

$$
H_{\mu}(X) \quad=_{\text {def }} \quad\left\{f \in H(X): f_{*} \mu=\mu\right\}
$$

is a closed subgroup and so is itself a Polish group.

In Akin [1999] we described a family of bad measures on a Cantor space $X$. This was an uncountable subset $\mathcal{M}^{*}$ of $\mathcal{M}_{X}$ such that for $\mu_{1}, \mu_{2} \in \mathcal{M}^{*}$ and $f \in H(X)$

$$
f_{*} \mu_{1}=\mu_{2} \quad \Longleftrightarrow \quad \mu_{1}=\mu_{2} \text { and } f=1_{X} .
$$

That is, no two distinct measures in $\mathcal{M}^{*}$ are homeomorphic and for each $\mu \in \mathcal{M}^{*}$, the group $H_{\mu}(X)$ is trivial.

In this paper we consider good measures on a Cantor space $X$. 


\section{Good measures on Cantor space}

We begin with a bit of algebra.

Let $S$ be a subset of the unit interval $I$. We call $S$ group-like if

$$
S=G \cap I
$$

for $G$ an additive subgroup of $\mathbb{R}$.

If $(2.1)$ holds with $G$ a $\mathbb{Q}$ vector subspace of $\mathbb{R}$ where $\mathbb{Q}$ is the field of rationals, then we call $S \mathbb{Q}$-like. If (2.1) holds with $G$ a subfield of $\mathbb{R}$ we will call $S$ field-like.

For subsets $A, B$ of $\mathbb{R}$ we will write $A B=\{a b: a \in A$ and $b \in B\}$ and $A+B=$ $\{a+b: a \in A$ and $b \in B\}$. In particular, if $\mathbb{Z}$ is the group of integers, then

$$
S+\mathbb{Z}=\{\alpha+n: \alpha \in S \text { and } n \in \mathbb{Z}\} .
$$

For $G$ an additive subgroup of $\mathbb{R}$ we will call a positive real number $\alpha$ a divisor of $G$ if

$$
\alpha G=G
$$

That is, $\alpha \beta \in G$ iff $\beta \in G$. Let $\operatorname{Div}(G)$ denote the set of divsors of $G$. Clearly, $\operatorname{Div}(G)$ is a multiplicative subgroup of the multiplicative group $\mathbb{R}^{*}$ of positive reals. Furthermore,

$$
\mathbb{Z} \subset G \quad \Longrightarrow \quad \operatorname{Div}(G) \subset G .
$$

Lemma 2.1. Let $S$ be a subset of $I$ with $0,1 \in S$. Let $G[S]$ be the additive subgroup of $\mathbb{R}$ generated by $S$.

(a) $\quad S \subset S+\mathbb{Z} \subset G[S]$ and $\quad S=(S+\mathbb{Z}) \cap I$.

(b) The following conditions on $S$ are equivalent:

(1) $S$ is group-like.

(2) $S+\mathbb{Z}=G[S]$.

(3) $S+\mathbb{Z}$ is an additive subgroup of $\mathbb{R}$.

(4) $\alpha, \beta \in S$ and $\alpha \leq \beta$ imply that $\beta-\alpha \in S$.

If $S$ is group-like and $G$ is an additive subgroup of $\mathbb{R}$, then

$$
S=G \cap I \quad \Longleftrightarrow \quad S+\mathbb{Z}=G .
$$

(c) When $S$ is group-like we let $\operatorname{Div}(S)$ denote Div $(S+\mathbb{Z})$. For $\alpha \in I \backslash\{0\}$

$$
\alpha \in \operatorname{Div}(S) \quad \Longleftrightarrow \quad \alpha S=S \cap[0, \alpha] .
$$

(d) The following conditions on $S$ are equivalent:

(1) $S$ is $\mathbb{Q}$-like.

(2) $S+\mathbb{Z}$ is a $\mathbb{Q}$ vector subspace of $\mathbb{R}$.

(3) $S$ is group-like and every positive integer is a divisor of $S+\mathbb{Z}$.

When $S$ is $\mathbb{Q}$-like, then $\mathbb{Q} \cap I \subset S$.

(e) The following conditions on $S$ are equivalent:

(1) $S$ is field-like.

(2) $S+\mathbb{Z}$ is a subfield of $\mathbb{R}$.

(3) $S$ is $\mathbb{Q}$-like and every positive element of $S$ is a divisor of $S+\mathbb{Z}$.

(4) $S$ is $\mathbb{Q}$-like and for every $\alpha \in S$

$$
\alpha S=S \cap[0, \alpha] .
$$

(f) $S$ is field-like iff all of the following five properties hold:

(i) $\mathbb{Q} \cap I \subset S$.

(ii) $\alpha \in S \Longrightarrow 1-\alpha \in S$. 
(iii) $\quad \alpha, \beta \in S \Longrightarrow \alpha \beta,(\alpha+\beta) / 2 \in S$.

(iv) $\alpha, \beta \in S$ and $\alpha+\beta \neq 0 \Longrightarrow \alpha /(\alpha+\beta) \in S$.

(v) $\alpha \in S$ and $\alpha<1 / 2 \Longrightarrow 2 \alpha \in S$.

Proof. (a) Since $0,1 \in S$ the inclusions are clear. On the other hand, if $\alpha+n \in I$ with $\alpha \in S$ and $n \in \mathbb{Z} \backslash\{0\}$, then either $n=1$ and $\alpha=0$ or $n=-1$ and $\alpha=1$. Either way, $\alpha+n \in\{0,1\}$. Hence the equation follows.

(b) $(1) \Rightarrow(4)$ : Obvious.

(4) $\Rightarrow(3)$ : If $\alpha \leq \beta$ in $S$, then $\beta-\alpha \in S$ by (4) and $\alpha-\beta+1=1-(\beta-\alpha) \in S$ by (4) applied twice. It easily follows that $S+\mathbb{Z}$ is closed under subtraction and so is an additive subgroup of $\mathbb{R}$.

$(3) \Rightarrow(2)$ : Obvious from the inclusions in (a).

$(2) \Rightarrow(1)$ : By the equality in (a), (2.1) holds with $G=G[S]$.

In general, if $S=G \cap I$ for some subgroup $G$ of $\mathbb{R}$, then by (a) $\mathbb{Z}+(G \cap I)=$ $S+\mathbb{Z} \subset G$. On the other hand, if $1 \in G$ and $G$ is a subgroup of $\mathbb{R}$, then any element of $G$ is of the form $n+\alpha$ with $\alpha \in G \cap I$. That is, $G \subset \mathbb{Z}+(G \cap I)$.

(c) Multiplying the equation in (a) by any positive real $\alpha$ we get

$$
\alpha S=(\alpha(S+\mathbb{Z})) \cap[0, \alpha]
$$

If, in addition, $\alpha \in I$, then

$$
S \cap[0, \alpha]=(S+\mathbb{Z}) \cap I \cap[0, \alpha]=(S+\mathbb{Z}) \cap[0, \alpha] .
$$

Thus, for $\alpha \in \operatorname{Div}(S), \alpha(S+\mathbb{Z})=S+\mathbb{Z}$ implies $\alpha S=S \cap[0, \alpha]$.

Conversely, if $\alpha S=S \cap[0, \alpha]$, then (a) and (2.9) imply that $(S+\mathbb{Z}) \cap I=S=$ $\left(\alpha^{-1} S \cap I\right)=\left(\alpha^{-1}(S+\mathbb{Z}) \cap I\right)$. Since $\alpha^{-1}(S+\mathbb{Z})$ is an additive subgroup of $\mathbb{R}$ it follows from (2.5) that $S+\mathbb{Z}=\alpha^{-1}(S+\mathbb{Z})$ and so $\alpha^{-1} \in \operatorname{Div}(S)$ which implies $\alpha \in \operatorname{Div}(S)$.

(d) $(1) \Leftrightarrow(2)$ and $(2) \Rightarrow(3)$ : Obvious from (b).

$(3) \Rightarrow(2)$ : Since $S$ is group-like $S+\mathbb{Z}$ is an additive group. Since every positive integer is a divisor, it is a rational vector space.

Since $1 \in S,(2)$ implies $\mathbb{Q} \subset S+\mathbb{Z}$. Intersect with $I$ and apply the equation in (a) to complete the proof of (d).

(e) $(1) \Leftrightarrow(2)$ and $(2) \Rightarrow(3)$ : Obvious from (b).

(3) $\Rightarrow(4)$ : Obvious from (c).

$(4) \Rightarrow(2): G=S+\mathbb{Z}$ is a $\mathbb{Q}$ vector space and from (2.7) we obtain $\beta G \subset G$ for any $\beta \in S$ from which it easily follows that $G$ is closed under multiplication. Given $g_{1}, g_{2} \in G$ with $g_{2}>0$ write the ratio $g_{1} / g_{2}$ as $\beta+n$ with $n \in \mathbb{Z}$ and $1 \geq \beta>0$. We have $\beta=g_{3} / g_{2}$ with $g_{3}=g_{1}-n g_{2}$ a positive element of $G$. Choose a positive integer $N$ greater than both $g_{1}$ and $g_{3}$. Replacing $g_{k}$ by $g_{k} / N$ for $k=1,3$ we remain in the rational vector space $G$. So we can assume that $g_{1}, g_{3} \in G \cap I=S$. Apply (2.7) with $\alpha=g_{3}$ to get that $\beta \in S$. Hence the ratio $g_{1} / g_{2}=\beta+n \in G$. It follows that $G$ is a field.

(f) If $S$ is field-like, then conditions (i)-(v) obviously hold. Now assume conditions (i)-(v). We will prove that $G=S+\mathbb{Z}$ is a field.

Observe first that if $\alpha, \beta \in S$ and $\alpha+\beta \leq 1$, then (iii) and (v) imply that $\alpha+\beta \in S$. If $\alpha \leq \beta$, then $(1-\beta)+\alpha \leq 1$ and so by (ii), (iii) and (v) $(1-\beta)+\alpha \in S$. By (ii) $\beta-\alpha=1-((1-\beta)+\alpha) \in S$. From part (b) it follows that $S$ is group-like and $G$ is a group. By (iii) $S$ is closed under multiplication from which it easily follows that $G=S+\mathbb{Z}$ is closed under multiplication. From (i) it follows that $\mathbb{Q} \subset S+\mathbb{Z}$ and so $G$ is a rational vector space and $S$ is $\mathbb{Q}$-like. 
By (e) it suffices to prove (2.7) for a positive $\alpha$ in $S$. Since $S$ is closed under multiplication by (iii), $\alpha S \subset S \cap[0, \alpha]$. On the other hand, if $\beta \in S$ and $\beta \leq \alpha$, then

$$
\beta / \alpha=\beta /(\beta+(\alpha-\beta))
$$

and so $\beta / \alpha \in S$ by (iv). Thus, (2.7) holds and so $G$ is a field by (e).

We will call a positive integer $n$ a reciprocal for $S$ if $1 / n \in S$. Let $\operatorname{Rec}(S)$ denote the set of reciprocals in S. Clearly,

$$
\operatorname{Div}(S) \cap \mathbb{Z}_{+} \quad \subset \quad \operatorname{Rec}(S) .
$$

Lemma 2.2. Let $S$ be a group-like subset of $I$ with $0,1 \in S$.

If $m / n \in S$ with $m, n$ relatively prime positive integers, then $n \in \operatorname{Rec}(S)$. If $n \in \operatorname{Rec}(S)$ and $m \mid n$, then $m \in \operatorname{Rec}(S)$. If $m, n \in \operatorname{Rec}(S)$, then the least common multiple, $\operatorname{lcm}(m, n)$, is in $\operatorname{Rec}(S)$.

In general,

$$
\mathbb{Q} \cap S=\{k / n: k=0,1, \ldots, n \text { and } n \in \operatorname{Rec}(S)\} .
$$

If $\operatorname{Rec}(S)$ is infinite, then $\mathbb{Q} \cap S$ is dense in I. If $\operatorname{Rec}(S)$ is finite, then $\mathbb{Q} \cap S$ is finite.

Proof. Observe that for $x, y \in \mathbb{Z}$ and positive integers $m, n$ :

$$
\begin{aligned}
x(m / n)+y(1) & =(x m+y n) / n, \\
x(1 / n)+y(1 / m) & =(x m+y n) / m n .
\end{aligned}
$$

Given such $m, n$ we can choose $x, y$ so that $x m+y n=\operatorname{gcd}(m, n)$, the greatest common divisor. So if $m / n \in S$ with $m, n$ relatively prime, then $1 / n \in S$ because $S$ is group-like. Equation (2.12) clearly follows and implies that any divisor of an element of $\operatorname{Rec}(S)$ is in $\operatorname{Rec}(S)$. Similarly, if the reciprocals of $m$ and $n$ are in $S$, then the second equation in (2.13) implies that $1 / \operatorname{lcm}(m, n)=\operatorname{gcd}(m, n) / m n \in S$. Finally, from (2.12) it is clear that if $\operatorname{Rec}(S)$ contains arbitrarily large integers, then $\mathbb{Q} \cap S$ is dense in $I$ and that otherwise the intersection is finite.

Remark. Notice that if $\operatorname{Rec}(S)$ is infinite and $S$ is group-like with $0,1 \in S$, then $\operatorname{Rec}(S)$ is directed with respect to the partial order of divisibility, i.e. $m, n \in \operatorname{Rec}(S)$ implies there exists $k \in \operatorname{Rec}(S)$ such that $m \mid k$ and $n \mid k$, namely $k=\operatorname{lcm}(m, n)$.

Definition 2.3. Let $X$ be a Cantor space. We say that a measure $\mu$ on $X$ satisfies the Subset Condition if whenever $U$ and $V$ are clopen subsets of $X$ with $\mu(U) \leq$ $\mu(V)$ there exists a clopen subset $U_{1}$ such that

$$
U_{1} \subset V \quad \text { and } \quad \mu\left(U_{1}\right)=\mu(U) \text {. }
$$

A measure $\mu$ on a Cantor space $X$ is called a good measure when $\mu \in \mathcal{M}_{X}$, i.e. it is full and nonatomic, and, in addition, $\mu$ satisfies the Subset Condition.

For a clopen subset $V$ of $X$ we relativize the clopen values set, defining

$$
S(\mu, V)={ }_{\text {def }} \quad\{\mu(U): U \text { is clopen in } X \text { and } U \subset V\} .
$$

Clearly, we always have

$$
S(\mu, V) \quad \subset \quad S(\mu) \cap[0, \mu(V)] .
$$


The Subset Condition says exactly that equality holds in (2.16). That is, $\mu$ satisfies the Subset Condition iff for every clopen subset $V$ of $X$

$$
S(\mu, V)=S(\mu) \cap[0, \mu(V)] .
$$

For a measure $\mu \in \mathcal{M}_{X}$ and $V$ a nonempty clopen subset of $X$ we have $\mu(V)>0$ and so we can define the measure $\mu_{V} \in \mathcal{M}_{V}$ by

$$
\mu_{V}(A) \quad=_{\text {def }} \quad \mu(A) / \mu(V)
$$

for every Borel subset $A \subset V$. We regard $\mu_{V}$ as a measure on $X$ by

$$
\mu_{V}(B) \quad=_{\text {def }} \quad \mu_{V}(B \cap V)=\mu(B \cap V) / \mu(V)
$$

for every Borel subset $B \subset X$. Regarded as a measure on $X, \mu_{V}$ is nonatomic but not full if $V$ is a proper subset of $X$.

Clearly, we have

$$
S\left(\mu_{V}\right)=(1 / \mu(V)) S(\mu, V) .
$$

It follows from (2.17) that $\mu$ is a good measure iff $\mu \in \mathcal{M}_{X}$ and for every nonempty clopen subset $V$ of $X$

$$
S\left(\mu_{V}\right)=[(1 / \mu(V)) S(\mu)] \cap I .
$$

Proposition 2.4. If $\mu$ is a good measure on a Cantor space $X$, then the clopen values set $S(\mu)$ is group-like. If, in addition, $V$ is a nonempty clopen subset of $X$, then $\mu_{V}$ is a good measure on the Cantor space $V$ and $S\left(\mu_{V}\right)$ is group-like.

Proof. Assume $\alpha=\mu(U) \leq \mu(V)=\beta$ for clopen subsets $U, V$. Apply the Subset Condition to get a clopen $U_{1}$ which satisfies (2.14). Since $V \backslash U_{1}$ is clopen,

$$
\beta-\alpha=\mu\left(V \backslash U_{1}\right) \quad \in S(\mu) .
$$

From Lemma 2.1(b) it follows that $S(\mu)$ is group-like.

It is clear that $\mu_{V}$ is nonatomic and full on $V$. The Subset Condition for $\mu$ easily implies the Subset Condition for $\mu_{V}$ on $V$. Hence, $\mu_{V}$ is good and so its clopen values set is group-like.

Definition 2.5. Let $(X, \leq)$ be an ordered Cantor space and $\mu \in \mathcal{M}_{X}$. We say that $\mu$ is adapted to $(X, \leq)$, or that the order $\leq$ is adapted to $\mu$, when

$$
\tilde{S}(\mu)=S(\mu) \text {. }
$$

Theorem 2.6. Let $(X, \leq)$ be an ordered Cantor space and $\mu \in \mathcal{M}_{X}$.

(a) The measure $\mu$ is adapted to $(X, \leq)$ iff the special clopen values set $\tilde{S}(\mu)$ is a group-like subset of $I$.

(b) If $\mu$ is adapted to $(X, \leq)$, then $\mu$ satisfies the Subset Condition and so is a good measure on $X$.

(c) If $\mu$ is adapted to $(X, \leq)$ and $V$ is a nonempty clopen subset of $X$, then $\mu_{V}$ is adapted to the ordered Cantor space $(V, \leq)$, with the order induced from $(X, \leq)$.

Proof. (a) If $\tilde{S}(\mu)$ is group-like, then by Lemma 2.1(a),(b) $\tilde{S}(\mu)=G[\tilde{S}(\mu)] \cap I$ and so by (1.22) $\tilde{S}(\mu)=S(\mu)$. On the other hand, suppose that $0<\alpha \leq \beta \in \tilde{S}(\mu)$. There exist left endpoints $x, y$ in $X$ such that $\alpha=\mu([0, x])$ and $\beta=\mu([0, y])$. Since $\mu$ is full $x \leq y$ in $X$. Since $(x, y]$ is clopen we have $\beta-\alpha=\mu((x, y]) \in S(\mu)$. So if $\tilde{S}(\mu)=S(\mu)$, then $\beta-\alpha \in \tilde{S}(\mu)$ and so $\tilde{S}(\mu)$ is group-like by Lemma 2.1(b). 
(b) If $V$ is a nonempty clopen subset of $X$, then it can be written as a finite, disjoint union of clopen intervals:

$$
\begin{array}{r}
V=\bigcup_{i=1}^{k} V_{i} \quad \text { with } \quad V_{i}=\left[x_{i}^{+}, y_{i}^{-}\right] \\
\quad \text { such that } x_{i}^{+}<y_{i}^{-}<x_{i+1}^{+}
\end{array}
$$

and $x_{i}^{+}$is a right endpoint and $y_{i}^{-}$is a left endpoint for all $i$. Define $y_{0}^{+}=m$ and let

$$
W_{i} \quad=_{\text {def }} \quad\left[y_{i-1}^{+}, x_{i}^{-}\right] \quad \text { for } i=1, \ldots, k,
$$

where $x_{i}^{-}, x_{i}^{+}$and $y_{i}^{+}, y_{i}^{-}$are endpoint pairs. If $x_{1}^{+}=m$, then $W_{1}=\operatorname{def} \emptyset$.

Now let $U$ be a nonempty clopen subset of $X$ such that $0<\mu(U) \leq \mu(V)$. Define $r \in\{1, \ldots, k\}$ so that

$$
\sum_{j=1}^{r-1} \mu\left(V_{j}\right)<\mu(U) \leq \sum_{j=1}^{r} \mu\left(V_{j}\right)
$$

and let

$$
\tilde{\alpha}=\sum_{j=1}^{r} \mu\left(W_{j}\right)+\mu(U),
$$

so that

$$
\mu\left(\left[m, x_{r}^{-}\right]\right)<\tilde{\alpha} \leq \mu\left(\left[m, y_{r}^{-}\right]\right) .
$$

By (a), $\tilde{S}(\mu)=S(\mu)$ implies that $\tilde{S}(\mu)$ is group-like and so $\tilde{\alpha} \in S(\mu)=\tilde{S}(\mu)$. That is, $\tilde{\alpha}=\mu([m, z])$, where $z$ is a left endpoint of $X$. Clearly, $x_{r}^{-}<z \leq y_{r}^{-}$and so $x_{r}^{+} \leq z$. Hence,

$$
U_{1} \quad=_{\text {def }} \quad[m, z] \cap V=[0, z] \backslash \bigcup_{j=1}^{r} W_{j}
$$

satisfies $\mu\left(U_{1}\right)=\mu(U)$.

Thus, $\mu$ satisfies the Subset Condition.

(c) Let $\alpha$ be a positive element of $S\left(\mu_{V}\right)$ so that $\mu(V) \alpha=\mu(U)$ for $U$ some clopen subset of $V$. We can apply the construction of part (b) to get $U_{1}=[0, z] \cap V$ with $\mu(U)=\mu\left(U_{1}\right)$. Clearly, $U_{1}$ is a clopen subset of $V$ such that $x \in V$ and $x \leq z$ imply $x \in U_{1}$. So by definition of $\tilde{S}, \alpha=\mu_{V}\left(U_{1}\right)$ is an element of $\tilde{S}\left(\mu_{V}\right)$.

The main result of this section is a converse construction using partitions. A partition $\mathcal{A}$ of a Cantor space $X$ is a pairwise disjoint cover of $X$ by nonempty clopen subsets. By compactness a partition is finite. Giving $\mathcal{A}$ the discrete topology we define the carrier map as the continuous, locally constant surjection $\pi^{\mathcal{A}}: X \rightarrow \mathcal{A}$ given by

$$
x \in \pi^{\mathcal{A}}(x) \quad \text { for } \quad x \in X .
$$

If $d$ is a metric on $X$ and $\mu$ is a measure on $X$ we define the mesh of $\mathcal{A}$ and the $\mu$ mesh of $\mathcal{A}$ by

$$
\begin{aligned}
& \operatorname{mesh}(\mathcal{A})={ }_{\text {def }} \quad \max \{d(A): A \in \mathcal{A}\}, \\
& \mu \operatorname{mesh}(\mathcal{A}) \quad=_{\text {def }} \quad \max \{\mu(A): A \in \mathcal{A}\} .
\end{aligned}
$$


A partition $\mathcal{B}$ refines $\mathcal{A}$, written $\mathcal{B} \rightarrow \mathcal{A}$ if each element of $\mathcal{B}$ is contained in a, necessarily unique, member of $\mathcal{A}$. We define the surjection $\pi_{\mathcal{B}}^{\mathcal{A}}: \mathcal{B} \rightarrow \mathcal{A}$ by

$$
B \subset \pi_{\mathcal{B}}^{\mathcal{A}}(B) \quad \text { for } B \in \mathcal{A} \text {. }
$$

Clearly,

$$
\pi^{\mathcal{A}}=\pi_{\mathcal{B}}^{\mathcal{A}} \circ \pi^{\mathcal{B}} .
$$

Hence, if three partitions satisfy $\mathcal{C} \rightarrow \mathcal{B} \rightarrow \mathcal{A}$, then

$$
\pi_{\mathcal{C}}^{\mathcal{A}}=\pi_{\mathcal{B}}^{\mathcal{A}} \circ \pi_{\mathcal{E}}^{\mathcal{B}} .
$$

An ordered partition $(\mathcal{A}, \leq)$ is a partition with a total order on it. If $(\mathcal{A}, \leq)$ is an ordered partition, then we can order number $\mathcal{A}$. That is, write

$$
\mathcal{A}=\left\{A_{0}, \ldots, A_{n}\right\} \quad \text { with } i<j \quad \Leftrightarrow \quad A_{i}<A_{j} .
$$

If $(\mathcal{B}, \leq)$ and $(\mathcal{A}, \leq)$ are ordered partitions, then we write $(\mathcal{B}, \leq) \rightarrow(\mathcal{A}, \leq)$ if $\mathcal{B} \rightarrow \mathcal{A}$ and

$$
B_{1} \leq B_{2} \quad \Longrightarrow \quad \pi_{\mathcal{B}}^{\mathcal{A}}\left(B_{1}\right) \leq \pi_{\mathcal{B}}^{\mathcal{A}}\left(B_{2}\right) \quad \text { for } B_{1}, B_{2} \in \mathcal{B}
$$

Conversely, if $(\mathcal{A}, \leq)$ is an ordered partition and $\mathcal{B} \rightarrow \mathcal{A}$, then by choosing an arbitrary ordering on $\left(\pi_{\mathcal{B}}^{\mathcal{A}}\right)^{-1}(A)$ for each $A \in \mathcal{A}$ we obtain an ordering on $\mathcal{B}$ so that $(\mathcal{B}, \leq) \rightarrow(\mathcal{A}, \leq)$.

Theorem 2.7. Let $\mu$ be a good measure on a Cantor space $X$ and let $x_{0} \in X$. There exists an order $\leq$ on $X$ such that the minimum point $m$ of $(X, \leq)$ is $x_{0}$ and the measure $\mu$ is adapted to $(X, \leq)$, i.e. $\tilde{S}(\mu)=S(\mu)$.

Proof. Choose a metric $d$ on $X$. Write $S(\mu) \backslash\{0\}=\left\{\alpha_{0}, \alpha_{1}, \alpha_{2}, \ldots\right\}$ with $\alpha_{0}=1$.

For each $k=0,1,2, \ldots$ we will construct an ordered partition $\left(\mathcal{A}_{k}, \leq\right)$ and order number it: $\mathcal{A}_{k}=\left\{A_{k 0}, \ldots, A_{k n_{k}}\right\}$ and we will define $j_{k} \leq n_{k}$ so that the following conditions hold:

(i) If $k \geq 1$, then $\left(\mathcal{A}_{k}, \leq\right) \rightarrow\left(\mathcal{A}_{k-1}, \leq\right)$.

(ii) $\operatorname{mesh}\left(\mathcal{A}_{k}\right) \leq 1 / k$ and $\mu \operatorname{mesh}\left(\mathcal{A}_{k}\right) \leq \alpha_{k}$.

(iii) $\pi^{\mathcal{A}_{k}}\left(x_{0}\right)$ is the minimum element $A_{k 0}$ of $\mathcal{A}_{k}$.

(iv) $\alpha_{k}=\sum\left\{\mu\left(A_{k j}\right): 0 \leq j \leq j_{k}\right\}$.

Begin with $\mathcal{A}_{0}=\{X\}$ and $j_{0}=0$. Since $\alpha_{0}=1$, conditions (i)-(iv) hold for $k=0$. Now proceed inductively assuming that $k>1$ and $\left(\mathcal{A}_{k-1}, \leq\right)$ has been defined.

Because the measure is nonatomic we can partition and order each $A_{(k-1) j}$ separately to obtain an ordered partition $(\mathcal{B}, \leq)$ which refines $\left(\mathcal{A}_{k-1}, \leq\right)$, has mesh at most $1 / k$ and $\mu$ mesh at most $\alpha_{k}$. We can choose the ordering on the partition of $A_{(k-1) 0}$ so that $x_{0}$ is in the minimum element. Thus, as a candidate for $\left(\mathcal{A}_{k}, \leq\right),(\mathcal{B}, \leq)$ satisfies conditions (i)-(iii). Order number $\mathcal{B}=\left\{B_{0}, \ldots, B_{n}\right\}$ and define $j *$ so that

$$
\sum\left\{\mu\left(B_{j}\right): 0 \leq j<j *\right\}<\alpha_{k} \leq \sum\left\{\mu\left(B_{j}\right): 0 \leq j \leq j *\right\} .
$$

Case i: If $\alpha_{k}=\sum\left\{\mu\left(B_{j}\right): 0 \leq j \leq j *\right\}$, then let $\left(\mathcal{A}_{k}, \leq\right)=(\mathcal{B}, \leq)$ and $j_{k}=j *$.

Case ii: If both inequalities are strict, then $\mu$ mesh $(\mathcal{B}) \leq \alpha_{k}$ implies that $0<j *$. Because $S(\mu)$ is group-like $\tilde{\alpha}={ }_{\text {def }} \alpha_{k}-\sum\left\{\mu\left(B_{j}\right): 0 \leq j<j *\right\}$ is a member of $S(\mu)$ and so by the Subset Condition we can choose a clopen subset $U \subset B_{j *}$ with 
$\mu(U)=\tilde{\alpha}$. Replace $B_{j *}$ by the two clopen sets $U, B_{j *} \backslash U$. This defines the ordered partition $\left(\mathcal{A}_{k}, \leq\right) \rightarrow(\mathcal{B}, \leq)$ when listed in order $\mathcal{A}_{k}=\left\{B_{0}, \ldots, B_{j *-1}, U, B_{j *} \backslash\right.$ $\left.U, B_{j *+1}, \ldots, B_{n}\right\}$. Thus, $n_{k}=n+1$. If we let $j_{k}=j *$, then condition (iv) holds. Since $0<j *$ the minimum element is unchanged and so it still contains $x_{0}$.

This completes the inductive construction. Now define the order on $X$ by

$$
x \leq y \quad \Longleftrightarrow \quad \pi^{\mathcal{A}_{k}}(x) \leq \pi^{\mathcal{A}_{k}}(y) \text { for all } k .
$$

The relation is clearly reflexive, transitive and closed. Because $\left(\mathcal{A}_{k}, \leq\right)$ refines $\left(\mathcal{A}_{k-1}, \leq\right)$ for all $k$ any two elements are comparable. Since the mesh of $\mathcal{A}_{k}$ tends to zero the relation is anti-symmetric. Thus we have defined a total order on $X$. From condition (iii) it is clear that $x_{0}$ is the minimum element $m$ of $X$.

Finally, let $z_{k}$ be the maximum element of the clopen set $A_{k j_{k}}$. Clearly,

$$
\left[m, z_{k}\right]=\bigcup\left\{A_{k j}: 0 \leq j \leq j_{k}\right\} .
$$

Since the maximum of a nonempty clopen set is a left endpoint, condition (iv) implies that $\alpha_{k} \in \tilde{S}(\mu)$. As this is true for all $k$ we have $S(\mu) \subset \tilde{S}(\mu)$. By $(1.22)$ the reverse inclusion is always true. Hence, with this choice of order $\mu$ is adapted to $(X, \leq)$.

Corollary 2.8. Let $X$ be a Cantor space and $\mu$ be a full, nonatomic measure on $X$. The measure $\mu$ is good on $X$, i.e. it satisfies the Subset Condition, iff there exists an ordering $\leq$ on $X$ with respect to which $\tilde{S}(\mu)=S(\mu)$.

Proof. If such an ordering exists, then $\mu$ is good by Theorem 2.6(b). Conversely, if $\mu$ is good, then the required ordering exists by Theorem 2.7 .

Together with Theorem 2.6(b) this result yields Theorem 0.1 of the Introduction.

It also follows that for good measures the clopen values set is a complete invariant. We will call two measures on a space homeomorphic if there is a homeomorphism mapping one to the other. Similarly, we will call two measures on an ordered space order isomorphic if there is an order isomorphism mapping one to the other.

Theorem 2.9. For $k=1,2$ let $X_{k}$ be a Cantor space, $\mu_{k}$ be a measure on $X_{k}$ and $x_{k} \in X_{k}$. Assume that $\mu_{1}$ is a good measure on $X_{1}$.

(a) If there exists a homeomorphism $f: X_{1} \rightarrow X_{2}$ such that $f_{*} \mu_{1}=\mu_{2}$, then $\mu_{2}$ is a good measure on $X_{2}$ and $S\left(\mu_{1}\right)=S\left(\mu_{2}\right)$.

Conversely, if $\mu_{2}$ is a good measure on $X_{2}$ and $S\left(\mu_{1}\right)=S\left(\mu_{2}\right)$, then there exists a homeomorphism $f: X_{1} \rightarrow X_{2}$ such that $f_{*} \mu_{1}=\mu_{2}$. Furthermore, $f$ can be chosen so that $f\left(x_{1}\right)=x_{2}$.

In particular, two good measures are homeomorphic iff they have the same clopen values set.

(b) If there exists a continuous map $f: X_{1} \rightarrow X_{2}$ such that $f_{*} \mu_{1}=\mu_{2}$, then $f$ is surjective and $S\left(\mu_{1}\right) \supset S\left(\mu_{2}\right)$.

Conversely, if $\mu_{2}$ is a good measure on $X_{2}$ and $S\left(\mu_{1}\right) \supset S\left(\mu_{2}\right)$, then there exists a continuous, almost one-to-one surjection $f: X_{1} \rightarrow X_{2}$ such that $f_{*} \mu_{1}=\mu_{2}$. Furthermore, $f$ can be chosen so that $f\left(x_{1}\right)=x_{2}$.

Proof. (a) It is easy to check that the absence of atoms, fullness and the Subset Condition are homeomorphism invariants. The clopen values set is a homeomorphism invariant by Proposition 1.5. 
Conversely, if $\mu_{k}$ is good on $X_{k}$, then by Theorem 2.7 we can choose an ordering so that $\mu_{k}$ is adapted to $\left(X_{k}, \leq\right)$ and $x_{k}$ is the minimum point of $X_{k}$. If the clopen values sets agree, then $\tilde{S}\left(\mu_{1}\right)=S\left(\mu_{1}\right)=S\left(\mu_{2}\right)=\tilde{S}\left(\mu_{2}\right)$. By Theorem 1.6 there is a unique order isomorphism $f:\left(X_{1}, \leq\right) \rightarrow\left(X_{2}, \leq\right)$ such that $f_{*} \mu_{1}=\mu_{2}$. Such an order isomorphism maps the minimum point $x_{1}$ to the minimum point $x_{2}$.

(b) As in (a) the first part follows from Proposition 1.5. For the second we choose orderings as in (a) and apply Theorem 1.6 again.

We can rephrase these results using the language of Theorem 1.7. We have let $\mathcal{D}$ denote the uncountable collection of countable dense subsets of $I$ which contain 0,1 . Let $\mathcal{D}_{g}, \mathcal{D}_{q}, \mathcal{D}_{f}$ denote the subcollections of group-like, $\mathbb{Q}$-like and field-like elements of $\mathcal{D}$. There are uncountably many distinct countable subfields of $\mathbb{R}$ and so each of these collections is uncountable.

Theorem 2.10. Let $(X, \leq)$ be an ordered Cantor space. The set of measures adapted to $(X, \leq)$ is $\tilde{S}^{-1}\left(\mathcal{D}_{g}\right)$. For measures $\mu_{1}, \mu_{2}$ adapted to $(X, \leq), \tilde{S}\left(\mu_{1}\right)=$ $\tilde{S}\left(\mu_{2}\right)$ implies that $\mu_{1}$ and $\mu_{2}$ are order isomorphic. On the other hand, $\tilde{S}\left(\mu_{1}\right) \neq$ $\tilde{S}\left(\mu_{2}\right)$ implies that $\mu_{1}$ and $\mu_{2}$ are not even homeomorphic.

Proof. The first result is a restatement of Theorem 2.6(a). By Theorem 1.6 the special clopen values sets are order isomorphism invariants. For adapted measures these are the same as the clopen values sets which are homeomorphism invariants by Proposition 1.5.

If $H$ is a subgroup of the automorphism group $H(Z)$ of a Polish space $Z$, then $H$ acts on $Z$. If $z \in Z$, then $H z=\operatorname{def}_{\text {de }}\{h(z): h \in H\}$ is the $H$ orbit of $z$, or, when $H$ is understood, just the orbit of $z$. Two points $z_{1}, z_{2} \in Z$ are called $H$ equivalent if they lie in the same $H$ orbit, i.e. there exists $h \in H$ such that $h\left(z_{1}\right)=z_{2}$. The action is called transitive if $Z$ consists of a single orbit, i.e. all points are $H$ equivalent. The action is called topologically transitive if there exists a dense orbit. That is, the set

$$
\operatorname{Trans}(H) \quad=_{\text {def }} \quad\{z \in Z: \overline{H z}=Z\}
$$

is nonempty. In that case, it is a dense $G_{\delta}$ subset of the Polish space $Z$. The action is called minimal if every orbit is dense, i.e. $\operatorname{Trans}(H)=Z$. Of course, transitive $\Rightarrow$ minimal $\Rightarrow$ topologically transitive.

From Theorem 2.9(a) we see that if $\mu$ is a good measure on a Cantor space $X$, then the group $H_{\mu}(X)$ of measure preserving homeomorphisms acts transitively on $X$. We will strengthen this result below.

First, we go back to Subset Condition itself. In it we did not assume that the move from the clopen set $U$ to the clopen $U_{1} \subset V$ was effected by a measure automorphism. That is, we did not assume that $U_{1}=h(U)$ for some $h \in H_{\mu}(X)$. In fact, we obtain this apparently stronger condition for free.

Proposition 2.11. Let $\mu$ be a good measure on the Cantor space $X$.

(a) Let $U, V$ be clopen subsets of $X$ with $x \in U$ and $y \in V$. If $\mu(U) \leq \mu(V)$, then there exists $h \in H_{\mu}(X)$ such that

$$
h(x)=y \quad \text { and } \quad h(U) \subset V .
$$

Furthermore, if $h \in H_{\mu}(X)$ satisfies $h(U) \subset V$, then $h(U)=V$ iff $\mu(U)=$ $\mu(V)$. 
(b) Let $V$ be a clopen subset of $X$ with $x \in V$. If $\alpha \in S(\mu) \cap(0, \mu(V)]$, then there exists $U$ a clopen subset of $X$ such that

$$
x \in U, \quad U \subset V, \text { and } \mu(U)=\alpha .
$$

Proof. (a) By the Subset Condition there exists a clopen $U_{1} \subset V$ with $\mu\left(U_{1}\right)=$ $\mu(U)$. Let $G=U \backslash\left(U \cap U_{1}\right)$ and $G_{1}=U_{1} \backslash\left(U \cap U_{1}\right)$. So $G$ and $G_{1}$ are disjoint clopens with the same measure.

By Proposition 2.4, the measures $\mu_{G_{1}}$ and $\mu_{G}$ are good and by (2.20) they have the same clopen values set. By Theorem 2.9(a) there is a homeomorphism $f: G \rightarrow G_{1}$ which maps $\mu_{G}$ to $\mu_{G_{1}}$. Define $h_{1} \in H_{\mu}(X)$ to be $f$ on $G, f^{-1}$ on $G_{1}$ and the identity on $X \backslash\left(G \cup G_{1}\right)$. Clearly, $h_{1}(U)=U_{1}$. Let $x_{1}=h_{1}(x) \in V$. Now apply Theorem 2.9(a) to the good measure $\mu_{V}$. There exists a measure preserving automorphism of $V$ which maps $x_{1}$ to $y$. Extend by the identity on $X \backslash V$ to obtain $h_{2} \in H_{\mu}(X)$. Finally, let $h=h_{2} \circ h_{1}$.

If $h \in H_{\mu}$ and $h(U) \subset V$, then $V \backslash h(U)$ is a clopen subset of $X$. Since $\mu$ is full this difference is empty iff it has measure zero. Thus, $h(U)=V$ iff $\mu(U)=\mu(V)$.

(b) By the subset condition there exists a clopen $U_{1} \subset V$ with $\mu\left(U_{1}\right)=\alpha$. Since $\alpha>0$ there exists $x_{1} \in U_{1}$. By Theorem 2.9(a) there exists a measure preserving automorphism $f$ of $V$ which maps $x_{1}$ to $x$. Let $U=f\left(U_{1}\right)$.

Corollary 2.12. Let $\mu$ be a good measure on the Cantor space $X$. If $\mathcal{A}=\left\{A_{1}, \ldots, A_{n}\right\}$ and $\mathcal{B}=\left\{B_{1}, \ldots, B_{n}\right\}$ are partitions of $X$ with $\mu\left(A_{i}\right)=\mu\left(B_{i}\right)$ for $i=1, \ldots, n$, then there exists $h \in H_{\mu}(X)$ such that

$$
h\left(A_{i}\right)=B_{i} \quad \text { for } \quad i=1, \ldots, n .
$$

Proof. By Proposition 2.11(a) there exists $h_{i} \in H_{\mu}(X)$ such that $h_{i}\left(A_{i}\right)=B_{i}$ for $i=1, \ldots, n$. Let $h=h_{i}$ on $A_{i}$ for $i=1, \ldots, n$.

We now prove a strong homogeneity result.

Theorem 2.13. Let $\mu$ be a good measure on the Cantor space $X$. If $\left\{x_{1}, \ldots, x_{n}\right\}$ and $\left\{y_{1}, \ldots, y_{n}\right\}$ are two lists of $n$ distinct points in $X$, then there exists $h \in H_{\mu}(X)$ such that $h\left(x_{i}\right)=y_{i}$ for $i=1, \ldots, n$.

In particular, $H_{\mu}(X)$ acts transitively on $X$.

Proof. Since $X$ is perfect we can compare each list to another list of $n$ distinct points and so assume that all $2 n$ points are distinct. We can then choose $2 n$ pairwise disjoint clopen sets $\left\{U_{1}, \ldots, U_{n}\right\}$ and $\left\{V_{1}, \ldots, V_{n}\right\}$ so that $x_{i} \in U_{i}$ and $y_{i} \in V_{i}$ for $i=1, \ldots, n$. By applying Proposition 2.11(b) we can shrink them if necessary so that they all have the same measure $\alpha>0$. Apply Proposition 2.11(a) to obtain a measure preserving homeomorphism $h_{i}: U_{i} \rightarrow V_{i}$ which maps $x_{i}$ to $y_{i}$ for $i=1, \ldots, n$. Define $h \in H_{\mu}(X)$ to be $h_{i}$ on $U_{i}, h_{i}^{-1}$ on $V_{i}$ for $i=1, \ldots, n$ and the identity on $X \backslash \bigcup_{i=1}^{n}\left(U_{i} \cup V_{i}\right)$.

I do not know if there exist measures $\mu$ in $\mathcal{M}_{X}$ which are not good but for which $H_{\mu}(X)$ acts transitively. However, with some assumptions on the clopen values sets we do obtain the converse result.

Lemma 2.14. Let $\mu \in \mathcal{M}_{X}$ with $X$ a Cantor space.

(a) If the action of $H_{\mu}(X)$ on $X$ is transitive, minimal or topologically transitive, then for every nonempty clopen subset $V$ of $X$ the action of $H_{\mu_{V}}(V)$ on $V$ satisfies the corresponding property. 
(b) Assume that for every nonempty clopen subset $V$ of $X, S\left(\mu_{V}\right)$ is group-like. If for every pair of clopen subsets $U, V$ of $X$ with $\mu(U) \leq \mu(V)$ there exist clopen subsets $U_{1}, \ldots, U_{n}$ of $V$ such that

$$
\sum_{i=1}^{n} \mu\left(U_{i}\right)=\mu(U),
$$

then $\mu$ is a good measure on $X$.

Proof. (a) It suffices to show that for $x, y \in V$, if $y$ is in the $H_{\mu}$ orbit of $x$, then it is in the $H_{\mu_{V}}$ orbit of $x$. It suffices to find $g \in H_{\mu}$ with $g(x)=y$ and $g=1_{X}$ on $X \backslash V$. If $x=y$ let $g=1_{X}$. Otherwise, if $f \in H_{\mu}$ with $f(x)=y$, we can choose a clopen set $U$ with $x \in U$ such that $U$ is disjoint from the clopen $f(U)$ and $U \cup f(U) \subset V$. Define $g$ to be $f$ on $U, f^{-1}$ on $f(U)$ and the identity elsewhere.

(b) For $i=1, \ldots, n, \mu_{V}\left(U_{i}\right)$ is in the group-like set $S\left(\mu_{V}\right)$. Since the sum $\mu(U) / \mu(V)$ is in $I$, it is in $S\left(\mu_{V}\right)$ and so $\mu(U) \in S(\mu, V)$ by $(2.20)$. Thus, there is a clopen subset $U_{1}$ of $V$ such that $\mu(U)=\mu\left(U_{1}\right)$, proving the Subset Condition.

Theorem 2.15. Let $\mu \in \mathcal{M}_{X}$ with $X$ a Cantor space. Assume that for every nonempty clopen subset $V$ of $X, S\left(\mu_{V}\right)$ is group-like. If $H_{\mu}(X)$ acts minimally on $X$, then $\mu$ is a good measure on $X$.

Proof. Assume we are given a pair of clopen subsets $U, V$ of $X$ with $\mu(U) \leq \mu(V)$. With $H=H_{\mu}(X), H x \cap V \neq \emptyset$ for every $x \in U$. So for every $x \in U$ there exists $h_{x} \in H$ and a clopen $U_{x} \subset U$ such that $x \in U_{x}$ and $h_{x}\left(U_{x}\right) \subset V$. Choose a finite list $\left\{U_{x_{1}}, \ldots, U_{x_{n}}\right\}$ covering $U$ and define for $i=1, \ldots, n$

$$
\tilde{U}_{i}=U_{x_{i}} \backslash \bigcup_{j=1}^{i-1} U_{x_{j}} \quad \text { and } \quad U_{i}=h_{x_{i}}\left(\tilde{U}_{i}\right) .
$$

Clearly, each $U_{i} \subset V$ and (2.44) holds. It follows from Lemma 2.14(b) that $\mu$ is a good measure.

Remark. I do not know whether the group-like assumption is redundant. That is, I do not know of any example where $H_{\mu}$ acts minimally, but the measure is not good. If one could prove that $H_{\mu}$ acts minimally $\Longrightarrow S(\mu)$ is group-like, then Lemma 2.14(a) would imply that $S\left(\mu_{V}\right)$ is group-like for every nonempty clopen $V$ and so from the theorem, $\mu$ would be good.

A pointed monothetic group is a pair $(\Gamma, g)$, where $\Gamma$ is a Polish group and $g$ is a topological generator of $\Gamma$, i.e. the cyclic group generated by $g$ is dense in $\Gamma$. It follows that $\Gamma$ is abelian.

A map $h:\left(\Gamma_{1}, g_{1}\right) \rightarrow\left(\Gamma_{2}, g_{2}\right)$ is a homomorphism if $h: \Gamma_{1} \rightarrow \Gamma_{2}$ is a continuous group homomorphism with $h\left(g_{1}\right)=g_{2}$. If there exists a homomorphism $h$ from $\left(\Gamma_{1}, g_{1}\right)$ to $\left(\Gamma_{2}, g_{2}\right)$, then it is unique. When it exists then there is a homomorphism in the other direction iff $h$ is an isomorphism. If the domain is compact, then $h$ is surjective.

Assume now that $(\Gamma, g)$ is a compact, zero-dimensional, pointed monothetic group with Haar measure denoted $\mu_{\Gamma}$. Haar measure is always full and it is nonatomic iff $\Gamma$ is infinite. If $\Gamma$ is finite with order $n$, then $(\Gamma, g)$ is isomorphic to $\left(\mathbb{Z}_{n}, 1\right)$, the cyclic group of integers $\bmod n$ with 1 the congruence class of the number 1 . If $\Gamma$ is infinite, then it is topologically homogeneous and not discrete 
and so it is a Cantor space. We will call $(\Gamma, g)$ a pointed Cantor group when it is an infinite, compact, zero-dimensional pointed monothetic group.

If $(\Gamma, g)$ is a pointed Cantor group and $G$ is a clopen subgroup of $\Gamma$, then the set $\Gamma / G$ of cosets is a partition of $\Gamma$ and so $G$ has finite index. The quotient $(\Gamma / G, g+G)$ is isomorphic to $\left(\mathbb{Z}_{n}, 1\right)$, where $n$ is the index of $G$, denoted hereafter ind $G$. Conversely, if there is a homomorphism from $(\Gamma, g)$ to $\left(\mathbb{Z}_{n}, 1\right)$, then the kernel is a clopen subgroup of index $n$.

By starting with an ultrametric on the Cantor space $\Gamma$ and averaging using $\mu_{\Gamma}$, we can obtain an invariant ultrametric $d_{\Gamma}$ on $\Gamma$, i.e. if $g_{1}, g_{2}, g_{3} \in \Gamma$, then

$$
\begin{aligned}
& d_{\Gamma}\left(g_{1}, g_{3}\right) \leq \max \left(d_{\Gamma}\left(g_{1}, g_{2}\right), d_{\Gamma}\left(g_{2}, g_{3}\right)\right) \\
& \text { and } \quad d_{\Gamma}\left(g_{1}, g_{2}\right)=d_{\Gamma}\left(g_{1}+g_{3}, g_{2}+g_{3}\right) .
\end{aligned}
$$

If $\epsilon>0$ then the $d_{\Gamma}$ ball of radius $\epsilon$ about 0 is a clopen subgroup. Hence, the family $\mathcal{G}_{\Gamma}$ of clopen subgroups of $\Gamma$ is a countable base for the neighborhoods of 0 in $\Gamma$ and the collection of cosets of such clopen subgroups is a countable base for the topology of $\Gamma$. Clearly, $G_{1}, G_{2} \in \mathcal{G}_{\Gamma}$ implies $G_{1} \cap G_{2} \in \mathcal{G}_{\Gamma}$ with

$$
\text { lcm }\left(\text { ind } G_{1} \text {, ind } G_{2}\right) \mid \text { ind } G_{1} \cap G_{2} \mid \text { ind } G_{1} \cdot \text { ind } G_{2} \text {. }
$$

Define the set of positive integers

$$
D_{\Gamma} \quad{ }_{\text {def }} \quad\left\{\text { ind } G: G \in \mathcal{G}_{\Gamma}\right\} .
$$

We call a set $D$ of positive integers a divisibility set if

$$
m \mid n \text { and } n \in D \Longrightarrow m \in D \text { and } m, n \in D \Longrightarrow l c m(m, n) \in D \text {. }
$$

Clearly, if $(\Gamma, g)$ is a pointed Cantor group, then $D_{\Gamma}$ is a divisibility set. In general, for $S \subset I$ with $0,1 \in S$, if $S$ is group-like, then $\operatorname{Div}(S) \cap \mathbb{Z}_{+}$is a divisibility set and by Lemma $2.2 \operatorname{Rec}(S)$ is a divisibility set.

Let $D$ be an infinite divisibility set. The $D$-indexed family $\left\{\left(\mathbb{Z}_{n}, 1\right): n \in D\right\}$ with the canonical homomorphism from $\left(\mathbb{Z}_{n}, 1\right)$ to $\left(\mathbb{Z}_{m}, 1\right)$ when $m \mid n$ has as its inverse limit a pointed Cantor group denoted $\left(\Gamma_{D}, 1\right)$. It is called the adding machine associated with $D$. By counting $D$ and letting $m_{k}$ be the least common multiple of the first $k$ members of $D$ we can obtain a cofinal, increasing sequence $\left\{m_{k}: k=\right.$ $0,1 \ldots\}$ in $D, m_{k} \mid m_{k+1}$ for $k=0,1, \ldots$. Then $\left(\Gamma_{D}, 1\right)$ is isomorphic to the inverse limit of the sequence of projections $\left\{\left(\mathbb{Z}_{m_{k+1}}, 1\right) \rightarrow\left(\mathbb{Z}_{m_{k}}, 1\right): k=0,1, \ldots\right\}$.

If $D=D_{\Gamma}$ for any pointed Cantor group $(\Gamma, g)$, then the homomorphisms $(\Gamma, g) \rightarrow(\Gamma / G, g+G) \cong\left(\mathbb{Z}_{n}, 1\right)$, where $G \in \mathcal{G}_{\Gamma}$ and $i n d G=n$, induce an isomorphism from $(\Gamma, g)$ onto the adding machine for $D_{\Gamma}$.

If $S$ is a group-like subset of $I$ with $0,1 \in S$ such that $\operatorname{Rec}(S)$ is infinite, or, equivalently by Lemma $2.2, \mathbb{Q} \cap S$ is dense in $I$, then we call $\left(\Gamma_{\operatorname{Rec}(S)}, 1\right)$ the adding machine associated with $S$.

Theorem 2.16. Let $(\Gamma, g)$ be a pointed Cantor group. If $\mu_{\Gamma}$ is Haar measure on $\Gamma$, then $\mu_{\Gamma}$ is a good measure on $\Gamma$ with

$$
S\left(\mu_{\Gamma}\right)=\left\{k / n: k=0,1, \ldots, n \text { and } n \in D_{\Gamma}\right\} .
$$

Conversely, if $\mu$ is a good measure on a Cantor space $X$ and $S(\mu) \subset \mathbb{Q}$, then with $\Gamma=\Gamma_{R e c(S)}$ there is a homeomorphism $h: X \rightarrow \Gamma$ such that $h_{*} \mu=\mu_{\Gamma}$. That is, any good measure with clopen values set contained in the rationals is homeomorphic to the Haar measure on a pointed Cantor group. Furthermore, the measure $\mu$ uniquely determines the group up to isomorphism of pointed Cantor groups. 
Proof. The cosets of clopen subgroups of $\Gamma$ form a basis for topology of $\Gamma$. Hence, if $U, V$ are clopens in $\Gamma$ there exists $G \in \mathcal{G}_{\Gamma}$ so that both $U$ and $V$ are unions of cosets of $G$. That is, if $n=$ ind $G$ and $\pi$ is the associated homomorphism onto $\Gamma / G \cong \mathbb{Z}_{n}$, then there exist finite $R, S \subset \mathbb{Z}_{n}$ such that $U=\pi^{-1}(R)$ and $V=\pi^{-1}(S)$. Because $\pi$ maps $\mu_{\Gamma}$ onto the uniform measure on $\mathbb{Z}_{n}$ we have

$$
\mu_{\Gamma}(U)=\# R / n \text { and } \mu_{\Gamma}(V)=\# S / n,
$$

where $\# R$ is the cardinality of $R$. From this equation (2.50) is clear. Also if $\mu(U) \leq \mu(V)$, then there exists $R_{1} \subset S$ such that $\# R=\# R_{1}$ and so $\pi^{-1}\left(R_{1}\right)$ is a clopen subset of $V$ with the same measure as $U$. This verifies the Subset Condition and so $\mu_{\Gamma}$ is good.

Conversely, if $\mu$ is a good measure and $S(\mu)=S(\mu) \cap \mathbb{Q}$, then by Lemma 2.2 and (2.50) the good measures $\mu$ and $\mu_{\Gamma}$ with $\Gamma=\Gamma_{R e c(S)}$ have the same clopen values sets. In particular, since $S(\mu)$ is dense in $I$, Lemaa 2.2 implies that $\operatorname{Rec}(S)$ is infinite $S(\mu)=S(\mu) \cap \mathbb{Q}$. By Theorem 2.9(a) they are homeomorphic measures.

Finally, if two pointed Cantor groups have homeomorphic Haar measures, then they have a common clopen values set $S$ and so each is isomorphic to $\left(\Gamma_{\operatorname{Rec}(S)}, 1\right)$ in the category of pointed Cantor groups.

Theorem 2.17. Let $\left(\Gamma_{1}, g_{1}\right)$ and $\left(\Gamma_{2}, g_{2}\right)$ be pointed Cantor groups with Haar measures $\mu_{\Gamma_{1}}$ and $\mu_{\Gamma_{2}}$, respectively. The following conditions are equivalent:

(1) $S\left(\mu_{\Gamma_{1}}\right) \supset S\left(\mu_{\Gamma_{2}}\right)$.

(2) $D_{\Gamma_{1}} \supset D_{\Gamma_{2}}$.

(3) There exists a continuous map $f: \Gamma_{1} \rightarrow \Gamma_{2}$ such that $f_{*} \mu_{\Gamma_{1}}=\mu_{\Gamma_{2}}$.

(4) There exists a continuous, almost one-to-one surjection $f: \Gamma_{1} \rightarrow \Gamma_{2}$ such that $f_{*} \mu_{\Gamma_{1}}=\mu_{\Gamma_{2}}$.

(5) There exists $h:\left(\Gamma_{1}, g_{1}\right) \rightarrow\left(\Gamma_{2}, g_{2}\right)$ a homomorphism of pointed Cantor groups.

Any homomorphism $h:\left(\Gamma_{1}, g_{1}\right) \rightarrow\left(\Gamma_{2}, g_{2}\right)$ of pointed Cantor groups is surjective and satisfies $h_{*} \mu_{\Gamma_{1}}=\mu_{\Gamma_{2}}$.

Proof. (1) $\Leftrightarrow(3) \Leftrightarrow$ (4) follow from Theorem 2.9(b) because the Haar measures on pointed Cantor groups are good.

$(1) \Leftrightarrow(2)$ By $(2.50) D_{\Gamma}$ determines $S\left(\mu_{\Gamma}\right)$. On the other hand, by (2.50) and (2.12), $D_{\Gamma}=\operatorname{Rec}\left(S\left(\mu_{\Gamma}\right)\right)$ and so $D_{\Gamma}$ is determined by $S\left(\mu_{\Gamma}\right)$.

$(2) \Leftrightarrow(5)$ If $h:\left(\Gamma_{1}, g_{1}\right) \rightarrow\left(\Gamma_{2}, g_{2}\right)$ is a homomorphism, then $G \in \mathcal{G}_{\Gamma_{1}}$ implies $h^{-1}(G) \in \mathcal{G}_{\Gamma_{2}}$ with ind $G=$ ind $h^{-1}(G)$. Consequently, $D_{\Gamma_{1}} \supset D_{\Gamma_{2}}$. On the other hand, from the adding machine construction expressing $(\Gamma, 1)$ as the inverse limit of the finite groups $\left\{(\Gamma / G, 1+G): G \in \mathcal{G}_{\Gamma}\right\}$, it is easy to see that the inclusion of (2) implies that the homomorphism $h:\left(\Gamma_{1}, g_{1}\right) \rightarrow\left(\Gamma_{2}, g_{2}\right)$ exists.

If $h:\left(\Gamma_{1}, g_{1}\right) \rightarrow\left(\Gamma_{2}, g_{2}\right)$ is a homomorphism, then because $h$ is surjective and $\mu_{\Gamma_{1}}$ is translation invariant it follows that $h_{*} \mu_{\Gamma_{1}}$ is translation invariant and so is the unique translation invariant measure $\mu_{\Gamma_{2}}$ on $\Gamma_{2}$.

We will call $(\Xi, g)$ the pointed Cantor group with $D_{\Xi}$ the entire set of positive integers, i.e. the pointed Cantor group with clopen subgroups of every positive index. We call $(\Xi, g)$ the universal adding machine. By (2.50) and Theorem 2.16, the Haar measure $\mu_{\Xi}$ is characterized up to homeomorphism as the unique good measure with clopen values set

$$
S\left(\mu_{\Xi}\right)=\mathbb{Q} \cap I .
$$


For each prime $p$ the ring of $p$-adic integers $\Gamma_{p}$ is the inverse limit of the sequence of projections $\mathbb{Z}_{p^{k+1}} \rightarrow \mathbb{Z}_{p^{k}}$. The generator $g_{p}$ is the sequence of congruence classes of 1 . The pointed additive group $\left(\Gamma_{p}, g_{p}\right)$ is a pointed Cantor group and we denote by $\mu_{p}$ the associated Haar measure. Clearly,

$$
D\left(\Gamma_{p}\right)=\left\{p^{i}: i=0,1,2, \ldots\right\}
$$

and so from $(2.50)$

$$
S\left(\Gamma_{p}\right)=\left\{k / p^{i}: 0 \leq k \leq p^{i} \text { and } i=0,1,2, \ldots\right\} .
$$

The topological group $\Xi$ is the product of copies of $\Gamma_{p}$ as the index $p$ varies over all primes.

If $[n]=\{1, \ldots, n\}$ with $n>1$ and $w:[n] \rightarrow I \backslash\{0,1\}$ is a positive distribution, i.e. $\sum w_{i}=1$, then the associated Bernoulli measures, denoted $\beta\left(w_{1}, \ldots, w_{n}\right)$, on $[n]^{\mathbb{Z}_{+}}$ and $[n]^{\mathbb{Z}}$ are the unique probability measures such that the projections to different factors are independent and the projection to each factor induces the distribution $w$ on $[n]$. Any bijection between $\mathbb{Z}_{+}$and $\mathbb{Z}$ induces a homeomorphism between these two. The Bernoulli measures are full and nonatomic and on $X=[n]^{\mathbb{Z}}$ the shift homeomorphism $s$ defined by $s(x)_{i}=x_{i+1}$ preserves the Bernoulli measure.

By using the base $p$ expansion of integers it is easy to obtain a homeomorphism from $\Gamma_{p}$ to $[p]^{\mathbb{Z}_{+}}$which maps $\mu_{p}$ to the uniform Bernoulli measure $\beta(1 / p, \ldots, 1 / p)$. In particular, on $[3]^{\mathbb{Z}_{+}}$the Bernoulli measure $\beta(1 / 3,1 / 3,1 / 3)$ is a good measure with clopen values set $\left\{k / 3^{i}: 0 \leq k \leq 3^{i}\right.$ and $\left.i=0,1,2, \ldots\right\}$. We conclude this section with an example which shows how close a bad measure can get to a good one. Compare the results to those of Theorem 2.15.

Theorem 2.18. If on $X=[2]^{\mathbb{Z}_{+}}$the measure $\mu$ is the Bernoulli measure $\beta(1 / 3,2 / 3)$, then $\mu \in \mathcal{M}_{X}$ with $S(\mu)=S\left(\mu_{3}\right)=\left\{k / 3^{i}: 0 \leq k \leq 3^{i}\right.$ and $\left.i=0,1,2, \ldots\right\}$. Furthermore, for every nonempty clopen subset $V$ of $X, S\left(\mu_{V}\right)$ is group-like and the action of the group $H_{\mu}(X)$ on $X$ is topologically transitive. On the other hand, if $e$ is the point in $X$ with $e_{i}=1$ for all $i \in \mathbb{Z}_{+}$, then $f(e)=e$ for all $f \in H_{\mu}(X)$, i.e. $e$ is a fixed point for the action of the group. In particular, $\mu$ is not a good measure on $X$.

Proof. The computation of $S(\mu)$ is given in Proposition 1.7 of Akin 1999. The computation of $S\left(\mu_{V}\right)$ for general clopen $V$ uses similar methods but is rather elaborate and so we will omit it here. The shift map $s$ on $[2]^{\mathbb{Z}}$ is topologically transitive and preserves the Bernoulli measure. Since the two versions of the Bernoulli measure are homeomorphic, it certainly follows that the action of $H_{\mu}(X)$ is topologically transitive. In fact, one can show that if for $x \in X$ both $x_{i}=1$ and $x_{i}=2$ for infinitely many $i$, then the $H_{\mu}$ orbit of $x$ is dense.

On the other hand, let $f$ be a homeomorphism on $X$ with $f(e) \neq e$. There exists a clopen set $V$ with $e \in V$ and $f(V)$ disjoint from $V$. There exists some positive integer $N$ and disjoint subsets $F_{1}, F_{2}$ of $[2]^{[N]}$ such that $V=\pi^{-1}\left(F_{1}\right)$ and $f(V)=\pi^{-1}\left(F_{2}\right)$, where $\pi:[2]^{\mathbb{Z}_{+}} \rightarrow[2]^{[N]}$ is the projection. Furthermore, $(1, \ldots, 1) \in F_{1}$ and so $(1, \ldots, 1) \notin F_{2}$. It follows that $\mu(V)=m_{1} / 3^{N}$ with $m_{1}$ odd while $\mu(f(V))=m_{2} / 3^{N}$ with $m_{2}$ even. Thus, $f$ is not measure preserving.

By Theorem 2.13, $H_{\mu}$ acts transitively on $X$ if $\mu$ is good. So in this case, $\mu$ is not good. 
Remark. In Proposition 1.7 of Akin [1999] we showed directly that $\beta(1 / 3,2 / 3)$ is not homeomorphic to $\beta(1 / 3,1 / 3,1 / 3)$. Since the clopen values set is a complete invariant for good measures, it follows that they cannot both be good. Since $\beta(1 / 3,1 / 3,1 / 3)$ is good, we see another reason why $\beta(1 / 3,2 / 3)$ cannot be good.

\section{THE FIELD-LIKE CASE}

Let $\mathbb{R}^{*}$ denote the multiplicative group of positive reals, with $\mathbb{Q}^{*}=\mathbb{R}^{*} \cap \mathbb{Q}$ and $\mathbb{Z}^{*}=\mathbb{R}^{*} \cap \mathbb{Z}$, so that $\mathbb{Q}^{*}$ is a subgroup and $\mathbb{Z}^{*}$ is at least closed under multiplication.

Recall that if $G$ is an additive subgroup of $\mathbb{R}$, then

$$
\operatorname{Div}(G) \quad=_{\text {def }} \quad\left\{\alpha \in \mathbb{R}^{*}: \alpha G=G\right\}
$$

is a multiplicative subgroup of $\mathbb{R}^{*}$. When $S$ is a group-like subset with $0,1 \in S$, we denote by $\operatorname{Div}(S)$ the multiplicative group $\operatorname{Div}(G)$, where $G=G[S]=S+\mathbb{Z}$. Clearly,

$$
S \text { is } \mathbb{Q} \text {-like } \Longleftrightarrow \mathbb{Q}^{*} \subset \operatorname{Div}(S) \Longleftrightarrow \mathbb{Z}^{*} \subset \operatorname{Div}(S) .
$$

Lemma 3.1. Let $\mu$ be a good measure on a Cantor space $X$ and let $V$ be a nonempty clopen subset of $X$.

$$
\operatorname{Div}\left(S\left(\mu_{V}\right)\right)=\operatorname{Div}(S(\mu)) .
$$

Furthermore, the following conditions are equivalent:

(1) $\mu(V) \in \operatorname{Div}(S(\mu))$.

(2) $S\left(\mu_{V}\right)=S(\mu)$.

(3) There exists a homeomorphism $h: V \rightarrow X$ such that

$$
h_{*} \mu_{V}=\mu .
$$

If $\leq i$ an ordering on $X$ which is adapted to $\mu$, then the above conditions imply that there is a unique order isomorphism $h:(V, \leq) \rightarrow(X, \leq)$ such that (3.4) holds.

Proof. Equivalence (2.6) implies that

$$
\alpha \in \operatorname{Div}(S) \quad \Longleftrightarrow \quad S=\left(\alpha^{-1} S\right) \cap I .
$$

By (2.21) and (2.5) the group generated by $S\left(\mu_{V}\right)$ equals $(\mu(V))^{-1}(S(\mu)+\mathbb{Z})$. Hence, $\alpha \in \operatorname{Div}(S(\mu))$ iff $\alpha \in \operatorname{Div}\left(S\left(\mu_{V}\right)\right)$, proving (3.3).

(1) $\Leftrightarrow(2)$ follows from (2.21) and (3.5).

$(2) \Leftrightarrow(3)$ follows from Theorem 2.9(a).

When the order on $X$ is adapted to $\mu$, then the order on $V$ is adapted to $\mu_{V}$ by Theorem 2.6(c). If condition (2) holds, then two two measures have the same special clopen values sets and so the unique order isomorphism $h$ exists by Theorem $1.6(\mathrm{~b})$.

Proposition 3.2. Let $\mu$ be a good measure on a Cantor space $X$. The multiplicative group Div $(S(\mu))$ is nontrivial iff there is a basis of clopen sets $U$ of $X$ such that $\mu_{U}$ on $U$ is homeomorphic to $\mu$ on $X$.

Proof. If $\alpha \in \operatorname{Div}(S(\mu)) \backslash\{1\}$, then 0 is a limit point of the subgroup $\left\{\alpha^{n}: n \in \mathbb{Z}\right\}$. If $x \in X, V \subset X$ is clopen and $\alpha \in \operatorname{Div}(S(\mu))$ with $\alpha \leq \mu(V)$, then by Proposition 2.11(b) there exists a clopen $U \subset V$ with $x \in U$ and $\mu(U)=\alpha$. Hence,

$$
\mathcal{U}={ }_{\text {def }} \quad\{U \subset X: U \text { is clopen and } \mu(U) \in \operatorname{Div}(S(\mu))\}
$$


is a basis for $X$. By Lemma 3.1 a clopen $U$ is in $\mathcal{U}$ iff $\mu_{U}$ is homeomorphic to $\mu$. Conversely, Lemma 3.1 implies that $\operatorname{Div}(S(\mu))$ is nontrivial if there is any proper clopen subset $U$ such that $\mu_{U}$ is homeomorphic to $\mu$.

Proposition 3.3. For $\mu$ a good measure on a Cantor space $X$ the following conditions are equivalent:

(1) $S(\mu)$ is $\mathbb{Q}$-like.

(2) $\mathbb{Q} \cap I \subset S(\mu)$ and for every nonempty clopen subset $U$ of $X$ with $\mu(U)$ rational, $\mu_{U}$ on $U$ is homeomorphic to $\mu$ on $X$.

(3) $\mathbb{Q} \cap I \subset S(\mu)$ and for every nonempty clopen subset $U$ of $X$ with $\mu(U)$ rational, $S\left(\mu_{U}\right)=S(\mu)$.

Proof. By Lemma 3.1 each of these conditions says exactly that $\mathbb{Q}^{*} \cap I \subset \operatorname{Div}(S(\mu))$.

Theorem 3.4. Let $X$ be a Cantor space and $\mu \in \mathcal{M}_{X}$. The following conditions on $\mu$ are equivalent:

(1) $\mu$ is a good measure on $X$ and $S(\mu)$ is field-like.

(2) $\mu$ is a good measure on $X$ and for every nonempty clopen subset $U$ of $X$, $\mu_{U}$ on $U$ is homeomorphic to $\mu$ on $X$.

(3) $S(\mu)$ is group-like and for every nonempty clopen subset $U$ of $X, S\left(\mu_{U}\right)=$ $S(\mu)$.

(4) For every nonempty clopen subset $U$ of $X, S\left(\mu_{U}\right)=S(\mu)$ and, in addition,

$$
(2 S(\mu)) \cap I \subset S(\mu) .
$$

Proof. (1) $\Rightarrow(2)$ If $S(\mu)$ is field-like, then for every nonempty clopen $U, \mu(U) \in$ $\operatorname{Div}(S(\mu))$. Apply Lemma 3.1.

$(2) \Rightarrow(3)$ Proposition 2.4 implies $S(\mu)$ is group-like and Proposition 1.5 implies that $S\left(\mu_{U}\right)=S(\mu)$.

$(3) \Rightarrow(4) S(\mu)$ group-like implies (3.7).

$(4) \Rightarrow(1)$ We first show that if for every nonempty clopen subset $U$ of $X S\left(\mu_{U}\right)=$ $S(\mu)$, then conditions (i)-(iv) of Lemma 2.1(f) hold.

If $\mu(U)=\alpha$, then $\mu(X \backslash U)=1-\alpha$. So condition (ii) holds for any $S(\mu)$.

If $\alpha, \beta \in S(\mu)$, then there exists a clopen $V$ with $\mu(V)=\alpha$. If $\alpha>0$, then $S\left(\mu_{V}\right)=S(\mu)$ and so there exists a clopen $U \subset V$ with $\mu_{V}(U)=\beta$ and so $\mu(U)=$ $\alpha \beta$. In particular, $S(\mu)$ is closed under multiplication.

Given a positive integer $n$ let $\left\{V_{1}, \ldots, V_{n}\right\}$ be a partition of cardinality $n$. As above we can find for $i=1, \ldots, n$ clopens $U_{i} \subset V_{i}$ such that $\mu\left(U_{i}\right)$ is the product $\mu\left(V_{1}\right) \ldots \mu\left(V_{n}\right)$. Let $U$ be the clopen $U_{1} \cup \ldots \cup U_{n}$. Clearly, $\left\{U_{1}, \ldots, U_{n}\right\}$ is a partition of $U$ with $\mu_{U}\left(U_{i}\right)=1 / n$ for $i=1, \ldots, n$. Hence, $k / n \in S\left(\mu_{U}\right)=S(\mu)$ for $k=0, \ldots, n$. So condition (i) holds.

Applying this construction with $n=2$ we can find two disjoint nonempty clopens $U_{1}, U_{2}$ with $\mu\left(U_{1}\right)$ and $\mu\left(U_{2}\right)$ a common positive number $\gamma$ and so with $U=U_{1} \cup$ $U_{2} \mu(U)=2 \gamma$. If $\alpha, \beta \in S(\mu)$, then there exist clopens $W_{1} \subset U_{1}$ and $W_{2} \subset U_{2}$ such that $\mu_{U_{1}}\left(W_{1}\right)=\alpha$ and $\mu_{U_{2}}\left(W_{2}\right)=\beta$. Let $W=W_{1} \cup W_{2}$. Clearly,

$$
\mu_{U}(W)=(\alpha+\beta) / 2 \quad \text { and } \quad \mu_{W}\left(W_{1}\right)=\alpha /(\alpha+\beta) .
$$

Since $S\left(\mu_{U}\right)=S(\mu)=S\left(\mu_{W}\right)$ this verifies conditions (iii) and (iv) of Lemma 2.1(f).

Assumption (3.7) implies that condition (v) holds as well. So by Lemma 2.1(f) $S(\mu)$ is field-like. It remains to verify the Subset Condition. 
Given clopens $U, V$ with $\mu(U)=\alpha<\beta=\mu(V)$ then $S(\mu)$ field-like implies that $\alpha / \beta \in S(\mu)=S\left(\mu_{V}\right)$ and so there exists a clopen $U_{1} \subset V$ such that $\mu_{V}\left(U_{1}\right)=\alpha / \beta$. Hence, $\mu\left(U_{1}\right)=\alpha=\mu(U)$.

Since the Subset Condition holds, $\mu$ is good on $X$.

Remark. While we need condition (3.7) for the proof, it may well be redundant. We have no examples which suggest otherwise.

Corollary 3.5. Let $(X, \leq)$ be an ordered Cantor space and $\mu \in \mathcal{M}_{X}$. If for every nonempty clopen subset $V$ of $X$

$$
S\left(\mu_{V}\right)=S(\mu)=\tilde{S}(\mu),
$$

then $\mu$ is good on $X$ and $S(\mu)$ is field-like.

Proof. By definition $\mu$ is adapted to $(X, \leq)$ and so by Theorem 2.6(b) $\mu$ is good on $X$. Thus, condition (3) of Theorem 3.4 holds and $(3) \Rightarrow(1)$ of the theorem yields the result.

Theorem 3.6. Let $\mu$ be a good measure on a Cantor space $X$ and let $\mu_{1}$ be a full measure on a zero-dimensional space $X_{1}$. The product space $X_{1} \times X$ is a Cantor space and the product measure $\mu_{1} \times \mu$ is full and nonatomic. If, in addition, we have

$$
S\left(\mu_{1}\right) \backslash\{0\} \quad \subset \quad \operatorname{Div}(S(\mu)),
$$

then there is a homeomorphism $h: X \rightarrow X_{1} \times X$ such that

$$
h_{*} \mu=\mu_{1} \times \mu \text {. }
$$

In particular, $\mu_{1} \times \mu$ is then a good measure on $X_{1} \times X$ with

$$
S(\mu)=S\left(\mu_{1} \times \mu\right) .
$$

Proof. Observe that $X_{1} \times X$ is a zero-dimensional space and it is perfect because $X$ is. Thus, the product is a Cantor space. Clearly, the product measure is full and it is nonatomic because $\mu$ is. To obtain the homeomorphism $h$ it suffices by Theorem 2.9(a) to prove directly that the product measure is good and that (3.12) holds.

Since the projection of $X_{1} \times X$ to $X$ maps $\mu_{1} \times \mu$ to $\mu$, it follows from (1.23) that $S(\mu) \subset S\left(\mu_{1} \times \mu\right)$. Any nonempty clopen $W$ in the product is a finite disjoint union of products $U_{i} \times V_{i}$ with $U_{i}$ clopen in $X_{1}$ and $V_{i}$ clopen in $X$ with $\alpha_{i}=\mu_{1}\left(U_{i}\right)>0$ and $\beta_{i}=\mu\left(V_{i}\right)>0$. Each $\beta_{i} \in S(\mu)$ and by assumption (3.10) each $\alpha_{i} \in \operatorname{Div}(S(\mu))$. Because $S(\mu)$ is group-like, $\mu(W)=\sum_{i} \alpha_{i} \beta_{i} \in S(\mu)$. Hence, (3.12) holds.

Because $\mu$ is good (2.17) implies that $S\left(\mu, V_{i}\right) \supset S(\mu) \cap\left[0, \beta_{i}\right]$ and so

$$
S\left(\mu_{1} \times \mu, U_{i} \times V_{i}\right) \quad \supset \quad \alpha_{i} S\left(\mu, V_{i}\right) \quad \supset \quad S(\mu) \cap\left[0, \alpha_{i} \beta_{i}\right],
$$

since $\alpha_{i}$ is a divisor of $S(\mu)+\mathbb{Z}$. If $\gamma<\sum_{i} \alpha_{i} \beta_{i}$, then there is a positive integer $r$ such that

$$
\sum_{i<r} \alpha_{i} \beta_{i}<\gamma \leq \sum_{i<r} \alpha_{i} \beta_{i}+\alpha_{r} \beta_{r}
$$

If, in addition, $\gamma \in S(\mu)$, then by equations (3.14) and (3.12), $\gamma-\sum_{i<r} \alpha_{i} \beta_{i}$ $\in S\left(\mu_{1} \times \mu, U_{r} \times V_{r}\right)$. It follows that $\gamma \in S\left(\mu_{1} \times \mu, W\right)$. Applying (2.16) with $V=W$ we obtain (2.17) for every clopen subset $W$. It follows that $\mu_{1} \times \mu$ satisfies the Subset Condition. 
Corollary 3.7. Assume that $\mu$ is a good measure on a Cantor space $X$ with $S(\mu)$ field-like. Let $Y$ be the product of a finite or countably infinite number of copies of $X$ and let $\nu$ be the product measure on $Y$ obtained with $\mu$ on each factor. The measure $\nu$ is good on the Cantor space $Y$ and there exists a homeomorphism $h: X \rightarrow Y$ such that

$$
h_{*} \mu=\nu .
$$

Proof. For $\mu \times \mu$ on $X \times X$ the result is Theorem 3.6 with $\mu_{1}=\mu$. For any finite number of copies the result follows by induction using Theorem 3.6 again.

If $Y$ is the product of copies of $X$ indexed by a countably infinite set $J$ and $U \subset V$ are clopen subsets of $Y$, then there exist a clopen subset $\tilde{U} \subset \tilde{V}$ of a subproduct indexed by some finite subset $F$ of $J$ such that $U$ and $V$ are the preimages of $\tilde{U}$ and $\tilde{V}$, respectively, via the projection map. It then follows from the finite product result that $S\left(\nu_{V}\right)=S(\mu)$. By (3) $\Rightarrow$ (1) of Theorem 3.4 it follows that $\nu$ is good on $Y$. That $h_{*} \mu=\nu$ then follows from Theorem 2.9(a).

\section{Automorphisms of GOOd measures}

In constructing automorphisms of a good measure it is useful to use an adapted ordering. As an illustration we have

Proposition 4.1. Let $(X, \leq)$ be an ordered Cantor space with $\mu \in \mathcal{M}_{X}$. Assume that

$$
\alpha \in \tilde{S}(\mu) \quad \Longrightarrow \quad 1-\alpha \in \tilde{S}(\mu),
$$

e.g. this holds if $\mu$ is adapted to $(X, \leq)$. There exists a unique $f \in H_{\mu}(X)$ which is order-reversing, i.e.

$$
x \leq y \quad \Longrightarrow \quad f(x) \geq f(y) .
$$

Proof. Let $\leq^{\prime}$ denote the reverse order on $X$. Since $\mu$ is nonatomic it is clear that $\alpha$ is in the special clopen values set for $\mu$ on $(X, \leq)$ iff $1-\alpha$ is in the special clopen values set for $\mu$ on $\left(X, \leq^{\prime}\right)$. By assumption these two sets are the same. So Theorem 1.6(b) implies there is a unique measure preserving order isomorphism from $(X, \leq)$ to $\left(X, \leq^{\prime}\right)$.

Remarks. (a) In general, $\alpha=\mu(U)$ implies that $1-\alpha=\mu(X \backslash U)$ and so it is always true that $\alpha \in S(\mu)$ implies $1-\alpha \in S(\mu)$. Hence, $S(\mu)=\tilde{S}(\mu)$ implies (4.1).

(b) If we let $F_{\mu}^{\prime}$ denote the $\mathrm{CDF}$ of $\mu$ with respect to the reverse order $\leq^{\prime}$, it is clear that

$$
F_{\mu}^{\prime}=1-F_{\mu} .
$$

So let us begin with $(X, \leq)$ an ordered Cantor space and a measure $\mu$ adapted to $(X, \leq)$, i.e. $\tilde{S}(\mu)=S(\mu)$. Recall from Proposition 1.3 that the CDF $F_{\mu}:(X, \leq$ )$\rightarrow(I, \leq)$ is an almost one-to-one, continuous surjection with $F_{\mu}\left(I \backslash I n j_{F_{\mu}}\right)=$ $S(\mu) \backslash\{0,1\}$. For $t \in S(\mu) \backslash\{0,1\}$ we let $t^{-}<t^{+}$denote the endpoint pair which is mapped to $t$ by $F_{\mu}$. For 0 , we will write $0^{-}=0^{+}=m$ and $1^{-}=1^{+}=M$, the minimum and maximum point, respectively. We will call a closed interval $J=\left[t_{1}, t_{2}\right]$ of $\mathbb{R}$ a $\mu$ interval if

$$
0 \leq t_{1}<t_{2} \leq 1 \quad \text { and } \quad t_{1}, t_{2} \in S(\mu),
$$


in which case we let

$$
\hat{J}={ }_{\text {def }}\left[t_{1}^{+}, t_{2}^{-}\right],
$$

so that $\hat{J}$ is the unique clopen interval which is mapped by $F_{\mu}$ onto $J$. Clearly,

$$
\begin{aligned}
F_{\mu}^{-1}(J) & =\hat{J} \cup\left\{t_{1}^{-}, t_{2}^{+}\right\}=\left[t_{1}^{-}, t_{2}^{+}\right], \\
F_{\mu}^{-1}\left(J^{\circ}\right) & =\hat{J} \backslash\left\{t_{1}^{+}, t_{2}^{-}\right\}=\left(t_{1}^{+}, t_{2}^{-}\right),
\end{aligned}
$$

where $J^{\circ}$ is the interior of $J$ in $\mathbb{R}$.

For $\alpha \in \mathbb{R}$ define the translation map $H_{\alpha}$ and the antitranslation map $H_{\alpha}^{-}$on $\mathbb{R}$ by

$$
H_{\alpha}(t)=t+\alpha \quad \text { and } \quad H_{\alpha}^{-}(t)=-t+\alpha .
$$

For a subinterval $J \subset \mathbb{R}$ let $|J|$ denote its length, which equals its Lebesgue measure $\lambda(J)$. If $J_{1}, J_{2}$ are closed subintervals of $\mathbb{R}$ with $\left|J_{1}\right|=\left|J_{2}\right|>0$, then there is a unique translation map $H_{21}^{+}$and a unique antitranslation map $H_{21}^{-}$such that

$$
H_{21}^{+}\left(J_{1}\right)=J_{2}=H_{21}^{-}\left(J_{1}\right) \text {. }
$$

Lemma 4.2. Assume that $(X, \leq)$ is an ordered Cantor space and $\mu$ is a measure adapted to $(X, \leq)$. Let $J_{1}$ and $J_{2}$ be $S(\mu)$ intervals with $\left|J_{1}\right|=\left|J_{2}\right|$. There exist unique measure preserving homeomorphisms $h_{21}^{+}, h_{21}^{-}: \hat{J}_{1} \rightarrow \hat{J}_{2}$ such that $h_{21}^{+}$is order-preserving and $h_{21}^{-}$is order-reversing. Furthermore, these are the unique continuous maps such that

$$
H_{21}^{ \pm} \circ F_{\mu}=F_{\mu} \circ h_{21}^{ \pm} .
$$

Proof. By Theorem 2.6(c) $\mu_{\hat{J}_{k}}$ is a measure adapted to $\left(\hat{J}_{k}, \leq\right)(k=1,2)$. By Theorem 2.6(a) they are good and so by $(2.21) \tilde{S}\left(\mu_{\hat{J}_{1}}\right)=\tilde{S}\left(\mu_{\hat{J}_{2}}\right)$. By Theorem 1.6 there is a unique order isomorphism $h_{21}^{+}$from $\mu_{\hat{J}_{1}}$ to $\mu_{\hat{J}_{2}}$ and it is the unique continuous map such that $F_{2} \circ h_{21}^{+}=F_{1}$, where $F_{k}$ is the CDF of $\mu_{\hat{J}_{k}}$ for $k=1,2$. The restriction of $F_{\mu}$ to $\hat{J}_{k}$ is $F_{k}$ composed with the order preserving linear map from $I$ to $J_{k}(k=1,2)$ and so the characterization (4.7) for $h_{21}^{+}$follows. For $h_{21}^{-}$ apply Proposition 4.1 and the Remarks thereafter.

By the circle we will mean the compact quotient group $\mathbb{R} / \mathbb{Z}$ with the projection homomorphism $\pi: \mathbb{R} \rightarrow \mathbb{R} / \mathbb{Z}$. $\pi$ restricts to a surjection $\pi: I \rightarrow \mathbb{R} / \mathbb{Z}$ which yields the circle as the space $I$ with 0 and 1 identified. We will call an element $\alpha \in \mathbb{R} / \mathbb{Z}$ rational (or irrational) if it is the projection under $\pi$ of a rational (resp. an irrational) real number.

If $F_{\mu}$ is the $\mathrm{CDF}$ of the measure $\mu$ we define the surjection $F_{\mu}^{\pi}$ and the subset $S^{\pi}(\mu)$ by

$$
\begin{aligned}
F_{\mu}^{\pi} & ={ }_{\text {def }} \quad \pi \circ F_{\mu}: X \rightarrow \mathbb{R} / \mathbb{Z}, \\
S^{\pi}(\mu) \quad=_{\text {def }} \quad F_{\mu}^{\pi}(S(\mu)) & =F_{\mu}^{\pi}(S(\mu)+\mathbb{Z}) \subset \mathbb{R} / \mathbb{Z} .
\end{aligned}
$$

For $\alpha \in \mathbb{R} / \mathbb{Z}$ define the translation map

$$
H_{\alpha}^{\pi}(t)=t+\alpha
$$

with addition in $\mathbb{R} / \mathbb{Z}$. 
Theorem 4.3. Assume that $(X, \leq)$ is an ordered Cantor space and $\mu$ is a measure adapted to $(X, \leq)$. $S^{\pi}(\mu)$ is a countable, dense subgroup of $\mathbb{R} / \mathbb{Z}$. For each $\alpha \in$ $S^{\pi}(\mu)$ there is a unique $h_{\alpha} \in H_{\mu}(X)$ such that

$$
F_{\mu}^{\pi} \circ h_{\alpha}=H_{\alpha}^{\pi} \circ F_{\mu}^{\pi} \text {. }
$$

Furthermore, the map $h_{\#}: S^{\pi}(\mu) \rightarrow H_{\mu}(X)$ defined by $\alpha \mapsto h_{\alpha}$ is a discontinuous group homomorphism.

Proof. As noted in (4.10), $S^{\pi}(\mu)$ is the image of the group $S(\mu)+\mathbb{Z}$ under the homomorphism $F_{\mu}^{\pi}$ and so it is a subgroup of the circle.

For $\alpha \in S(\mu) \backslash\{0,1\}$ consider the partitions $\left\{\left[m,(1-\alpha)^{-}\right],\left[(1-\alpha)^{+}, M\right]\right\}$ and $\left\{\left[\alpha^{+}, M\right],\left[m, \alpha^{-}\right]\right\}$of $X$. We use Lemma 4.2 to get two translation maps whose union is $h_{\alpha} \in H_{\mu}(X)$ which satisfies

$$
h_{\alpha}\left(\left[m,(1-\alpha)^{-}\right]\right)=\left[\alpha^{+}, M\right] \quad \text { and } \quad h_{\alpha}\left(\left[(1-\alpha)^{+}, M\right]\right)=\left[m, \alpha^{-}\right] \text {, }
$$

and which is order preserving on each piece. Equation (4.12) follows from (4.9).

We clearly have $H_{\alpha}^{\pi} \circ H_{\beta}^{\pi}=H_{\alpha+\beta}^{\pi}$ for $\alpha, \beta \in \mathbb{R} / \mathbb{Z}$. It follows from uniqueness of the lifting that $h_{\#}$ is a homomorphism. Since the homomorphism cannot be extended to the entire circle, it is not continuous.

We now consider the dynamic character of these maps. A homeomorphism $f$ on a space $X$ is called uniquely ergodic if it has a unique invariant measure. In the proposition below we collect the well-known results that we will need about such automorphisms.

First, we recall that if $G: X_{1} \rightarrow X_{2}$ is a continuous map, then it is called almost one-to-one if $\operatorname{Inj}_{G}=\left\{x \in X_{1}: G^{-1}(G(x))\right.$ is a singleton $\}$ is dense in $X_{1}$. By compactness $\left\{y \in X_{2}: d\left(G^{-1}(y)\right)<\epsilon\right\}$ is open for any positive $\epsilon$. Hence, $G\left(\operatorname{Inj}_{G}\right)$ and its preimage $I n j_{G}$ are $G_{\delta}$ sets. By Lemma 1.1(d) an almost one-to-one map is almost open.

Proposition 4.4. $\quad$ (a) Let $\mu$ be a full measure on a space $X$. If $f \in H_{\mu}(X)$ is uniquely ergodic, then $f$ is minimal on $X$. That is, for every $x \in X$ the orbit $\left\{f^{n}(x): n \in \mathbb{Z}\right\}$ is dense in $X$. Furthermore, $\mu$ is nonatomic or $X$ is finite.

(b) Let $(\Gamma, g)$ be a compact pointed monothetic group. The translation map $h_{g}$ on $\Gamma$ defined by $h_{g}(x)=g+x$ is uniquely ergodic with Haar measure $\mu_{\Gamma}$ the unique invariant measure.

(c) For $k=1,2$ let $\mu_{k}$ be a measure on a space $X_{k}$ and let $G: X_{1} \rightarrow X_{2}$ be a continuous map such that

$$
G_{*} \mu_{1}=\mu_{2} .
$$

If $\mu_{2}$ is nonatomic, then $\mu_{1}$ is nonatomic. If $\mu_{2}$ is full, then $G$ is surjective. In any case,

$$
\mu_{2}\left(X_{2} \backslash G\left(I n j_{G}\right)\right)=0 \quad \Longleftrightarrow \quad \mu_{1}\left(X_{1} \backslash I n j_{G}\right)=0 .
$$

We will say that $G$ induces a measure isomorphism when these conditions hold. If $\mu_{2}$ is full and $G$ induces a measure isomorphism, then $G$ is almost one-to-one iff $\mu_{1}$ is full.

If $\mu_{2}$ is nonatomic and $X_{2} \backslash G\left(\operatorname{Inj}_{G}\right)$ is countable, then $G$ induces a measure isomorphism. 
(d) For $k=1,2$ let $\mu_{k}$ be a measure on a space $X_{k}$ and $f_{k} \in H_{\mu_{k}}(X)$ and let $G: X_{1} \rightarrow X_{2}$ be a continuous map such that

$$
G \circ f_{1}=f_{2} \circ G \text {. }
$$

If $f_{2}$ is uniquely ergodic, then $G_{*} \mu_{1}=\mu_{2}$. If, in addition, $\mu_{2}$ is full and nonatomic and $G$ induces a measure isomorphism, then $G$ is an almost one-to-one surjection, $\mu_{1}$ is full and nonatomic and $f_{1}$ is uniquely ergodic.

Proof. (a) The restriction of $f$ to any minimal subset of $X$ admits an invariant measure. So if there is a unique invariant measure, then there is a unique closed invariant subset $M$ and the complement has measure zero. If the invariant measure is full, then $M=X$. If $x$ is an atom for an invariant measure $\mu$, then the orbit of $x$ consists of atoms with equal measure. Since the measure is finite, $x$ is a periodic point whose orbit must be all of $X$ since $f$ is minimal. That is, $X$ is finite or $\mu$ is nonatomic.

(b) Haar measure is the unique measure preserved by all translations and so it is $h_{g}$ invariant. On the other hand, if, for any measure $\mu$ on $\Gamma$, we define $\operatorname{Inv}_{\mu} \subset \Gamma$ to be those elements whose translation maps preserve $\mu$, then $I n v_{\mu}$ is a closed subgroup of $\Gamma$. If the generator $g \in \operatorname{Inv}_{\mu}$, then $\operatorname{Inv}_{\mu}=\Gamma$ and so $\mu$ is the Haar measure $\mu_{\Gamma}$.

(c) (4.14) implies that $G$ maps any atom of $\mu_{1}$ to an atom of $\mu_{2}$ and that $\mu_{2}\left(X_{2} \backslash G\left(X_{1}\right)\right)=0$. Hence, if $\mu_{2}$ is nonatomic, then $\mu_{1}$ is and if $\mu_{2}$ is full, then $X_{2} \backslash G\left(X_{1}\right)=\emptyset$. Since $\operatorname{Inj}_{G}=G^{-1}\left(G\left(\operatorname{Inj}_{G}\right)\right)$ (4.15) follows from (4.14). If the conditions of (4.15) hold, then for any closed subset $A$ of $X_{1}, G(A)$ is a closed subset of $X_{2}$ with $A \cap I n j_{G}=G^{-1}\left(G(A) \cap G\left(I n j_{G}\right)\right)$. Hence, (4.15) implies

$$
\mu_{1}(A)=\mu_{1}\left(A \cap I n j_{G}\right)=\mu_{2}\left(G(A) \cap G\left(I n j_{G}\right)\right)=\mu_{2}(G(A)) .
$$

Now if $\mu_{1}$ is full and $A$ has nonempty interior, then $\mu_{1}(A)>0$ implies $A$ meets $I n j_{G}$ and so $I n j_{G}$ is dense and $G$ is almost one-to-one. On the other hand, if $G$ is almost one-to-one, then by Lemma $1.1 G$ is almost open. So if $\mu_{2}$ is full and $A$ has nonempty interior, then $G(A)$ has nonempty interior and so $\mu_{2}(G(A))>0$ implies $\mu_{1}(A)>0$ and so $\mu_{1}$ is full.

The result when $G\left(\operatorname{Inj}_{G}\right)$ has a countable complement is obvious.

(d) If $\mu_{1}$ is an invariant measure for $f_{1}$, then (4.14) implies that $G_{*} \mu_{1}$ is an invariant measure for $f_{2}$. If $\mu_{2}$ is the unique invariant measure for $f_{2}$, then (4.14) holds. Now assume, in addition, that $\mu_{2}$ is full and nonatomic and $G$ induces a measure isomorphism. From (c) it follows that $G$ is an almost one-to-one surjection and that $\mu_{1}$ is full and nonatomic. From (4.17) it is clear that $\mu_{1}$ is determined by $\mu_{2}$ and so $f_{1}$ is uniquely ergodic.

Corollary 4.5. Let $(X, \leq)$ be an ordered Cantor space and let $\mu$ be a measure adapted to $(X, \leq)$. If $\alpha$ is an irrational element of $S^{\pi}(\mu)$, then the automorphism $h_{\alpha} \in H_{\mu}(X)$ is uniquely ergodic, where $h_{\alpha}$ is the lift under $F_{\mu}$ of the $\alpha$ translation map on the circle.

Proof. Since $\alpha$ is irrational, $(\mathbb{R} / \mathbb{Z}, \alpha)$ is a compact pointed monothetic group and so the translation map $H_{\alpha}^{\pi}$ is uniquely ergodic by Proposition 4.4(b). Since $F_{\mu}^{\pi}$ is injective on the complement of the countable set $F_{\mu}^{-1}(\tilde{S}(\mu))$, it induces a measure isomorphism from the full nonatomic measure $\mu$ on $X$ to the Haar measure, i.e. Lebesgue measure on the circle. By Proposition 4.4(d) the lift $h_{\alpha}$ is uniquely ergodic. 
Theorem 4.6. Let $X$ be a Cantor space. A measure $\mu \in \mathcal{M}_{X}$ is good on $X$, i.e. it satisfies the Subset Condition, iff there exists $f \in H_{\mu}(X)$ which is a uniquely ergodic homeomorphism on $X$.

Proof. Assume that $\mu$ is good. If $S(\mu) \subset \mathbb{Q}$, then by Theorem 2.16 the measure $\mu$ is homeomorphic to the Haar measure on the adding machine $\left(\Gamma_{D}, 1\right)$ with $D=$ $\operatorname{Rec}(S(\mu))$. By Proposition 4.4(b) the translation by 1 on $\Gamma$ is uniquely ergodic. On the other hand, if there exists an irrational in $S(\mu)$, then let $\alpha$ be the corresponding irrational element of $S^{\pi}(\mu)$. By Theorem 2.7 we can choose an order on $X$ adapted to $\mu$. By Corollary 4.5 the translation map $h_{\alpha}$ on $X$ is uniquely ergodic.

The converse result is due to Glasner and Weiss. Lemma 2.5 of Glasner and Weiss [1995] implies that if $f$ is a uniquely ergodic homeomorphism on a Cantor space $X$, then the invariant measure $\mu$ is good on $X$.

We have seen that if $\mu$ is a good measure on a Cantor space $X$ and $\alpha \in S^{\pi}(\mu)$, then there exists $f \in H_{\mu}(X)$ which has the $\alpha$ translation map of the circle as a factor. We do not know if this characterizes the set $S(\mu)$. That is, do there exist $\mu$ invariant maps on $X$ which factor over an $\alpha$ translation for any $\alpha$ not in $S^{\pi}(\mu)$ ?

It is possible to extend Theorem 2.16 to get a factorization result over adding machines.

Theorem 4.7. Assume that $\mu$ is a good measure on a Cantor space $X$ such that $D=\operatorname{Rec}(S(\mu))$ is infinite. Let $\left(\Gamma_{D}, 1\right)$ be the adding machine associated with $D$ with $h$ the 1 translation map on $\Gamma_{D}$. There exists $G: X \rightarrow \Gamma_{D}$ with $X \backslash$ Inj $_{G}$ countable and $f \in H_{\mu}(X)$ such that $G \circ f=h \circ G$. The homeomorphism $f$ on $X$ is uniquely ergodic.

Proof. We will sketch the proof leaving the details to the reader.

Choose an order on $X$ adapted to $\mu$. Let $F_{\mu}: X \rightarrow I$ be the CDF. We will use the inverse limit construction for $\Gamma_{D}$. To do so, let $\left\{m_{k}: k=0,1, \ldots\right\}$ be a strictly increasing sequence of positive integers, cofinal in $D$, with $m_{0}=1$ and $m_{k-1} \mid m_{k}$ for $k=1,2, \ldots$. For each $k=1,2, \ldots$ let $d_{k}$ be the positive integer $m_{k} / m_{k-1}$ so that $d_{k}>1$.

We will use the phrase "the $n$ pieces of the interval $[a, b]$ " to mean the intervals $\{[a+i d, a+(i+1) d]: i=0,1, \ldots, n-1\}$ with $d=(b-a) / n$ listed in ascending order.

Let $\left\{A_{1,1}, \ldots, A_{1, d_{1}-1}\right\}$ list the first $d_{1}-1$ of the $d_{1}$ pieces of $I$ excluding the last interval $A_{1}$. Similarly, let $\left\{B_{1,1}, \ldots, B_{1, d_{1}-1}\right\}$ list the last $d_{1}-1$ of the $d_{1}$ pieces of $I$ excluding the first interval $B_{1}$. The excluded intervals both have length $1 / m_{1}$.

Now let $\left\{A_{2,1}, \ldots, A_{2, d_{2}-1}\right\}$ list the first $d_{2}-1$ of the $d_{2}$ pieces of $A_{1}$ excluding the last interval $A_{2}$. Similarly, let $\left\{B_{2,1}, \ldots, B_{2, d_{1}-1}\right\}$ list the last $d_{2}-1$ of the $d_{2}$ pieces of $B_{1}$ excluding the first interval $B_{2}$. The excluded intervals both have length $1 / m_{2}$.

Continuing this inductive labeling we obtain two sequences of intervals $\left\{A_{k, j}\right\}$, $\left\{B_{k, j}\right\}$, converging to the points 1 and 0 , respectively, with $\left|A_{k, j}\right|=\left|B_{k, j}\right|=1 / m_{k}$. Since $D=\operatorname{Rec}(S(\mu))$ and $\mu$ is adapted to the order, the reciprocal of each $m_{k}$ lies in $\tilde{S}(\mu)$ and each of these is a $\mu$ interval. Because the intervals in each sequence are nonoverlapping, the lifted sequences $\left\{\hat{A}_{k, j}\right\}$ and $\left\{\hat{B}_{k, j}\right\}$ form a countable partition of $X \backslash\{M\}$ and $X \backslash\{m\}$, respectively. Use Lemma 4.2 to define $f$ on $\hat{A}_{k, j}$ as the unique order-preserving map to $\hat{B}_{k, j}$ and finally let $f(M)=m$. Clearly, $f \in H_{\mu}(X)$ 
and it is easy to check that it is a cyclic permutation of the lifts of the $m_{k}$ pieces of $I$. This provides the projection $G$ from $X$ to the inverse limit $\Gamma_{D}$ which maps $f$ to the translation by 1 .

Finally, the intervals provide an ordering on $\Gamma_{D}$ and it is easy to see that $G$ is the unique continuous map such that

$$
F_{\mu}=F_{\mu_{\Gamma}} \circ G \text {. }
$$

Hence, $I n j_{F_{\mu}} \subset I n j_{G}$ and so $X \backslash I n j_{G}$ is countable. It follows from Proposition $4.4(\mathrm{~d})$ that $f$ is uniquely ergodic.

Remark. Let $(\Gamma, g)$ be a pointed Cantor group. By Proposition 1.5 and (2.50) in Theorem 2.16 the measure $\mu$ can only be mapped to Haar measure $\mu_{\Gamma}$ when $S\left(\mu_{\Gamma}\right) \subset S(\mu) \cap \mathbb{Q}$ and so by Theorem 2.17 any such pointed Cantor group is a homomorphic image of $\left(\Gamma_{D}, 1\right)$ with $D=\operatorname{Rec}(S(\mu))$.

Theorem 4.8. Let $\mu$ be a good measure on a Cantor space $X$ such that $S(\mu)$ is field-like. Assume that $I \subset S(\mu)$ is such that $I \cup\{1\}$ is linearly independent over $\mathbb{Q}$ and let $\pi(I)$ be the corresponding subset of $S^{\pi}(\mu) \subset \mathbb{R} / \mathbb{Z}$. Let $H=\prod_{\alpha \in \pi(I)} H_{\alpha}^{\pi}$ be the translation map on the torus $\mathbf{T}_{I}$ (the product of copies of the circle $\mathbb{R} / \mathbb{Z}$ indexed by $\pi(I))$.

There exists $h \in H_{\mu}(X)$ and an almost one-to-one measure isomorphism $G$ : $X \rightarrow \mathbf{T}_{I}$ such that $G \circ h=H \circ G$. G maps $\mu$ to the Haar measure on $\mathbf{T}_{I}$ and the homeomorphism $h$ on $X$ is uniquely ergodic.

Proof. Let $g \in \mathbf{T}_{I}$ with $g_{\alpha}=\alpha$. The rational independence of $I \cup\{1\}$ implies that $\left(\mathbf{T}_{I}, g\right)$ is a compact pointed monothetic group and that $H$ is the $g$ translation map. By Proposition 4.4(b) $H$ is uniquely ergodic.

Let $Y$ be the product of copies of $X$ indexed by $\pi(I)$ with $\nu$ the measure on $Y$ which is the product of copies of $\mu$. Let $\tilde{G}: Y \rightarrow \mathbf{T}_{I}$ be the product of copies of $F_{\mu}^{\pi}: X \rightarrow \mathbb{R} / \mathbb{Z} . \operatorname{Inj}_{G}$ is the product of the dense sets $I n j_{F_{\mu}}$ and so it is dense, i.e. $\tilde{G}$ is an almost one-to-one surjection. The complement is the union of sets indexed by $\pi(I)$, where the points of the $\alpha$ set have $\alpha$ coordinate in $X \backslash I n j_{F_{\mu}}$. Hence, $Y \backslash I n j_{\tilde{G}}$ has measure zero, i.e. $\tilde{G}$ is a measure isomorphism. Let $\tilde{h} \in H_{\nu}(Y)$ be the product of copies of $h_{\alpha}$ from Theorem 4.3 so that $\tilde{G} \circ \tilde{h}=H \circ \tilde{G}$. By Proposition $4.4(\mathrm{~d}) \tilde{h}$ is uniquely ergodic.

Because $S(\mu)$ is field-like, Corollary 3.7 implies there exists a homeomorphism $q: X \rightarrow Y$ which maps $\mu$ to $\nu$. We let $h=q^{-1} \circ \tilde{h} \circ q$ and $G=\tilde{G} \circ q$.

Theorem 4.9. Let $\mu$ be a good measure on a Cantor space $X$ such that $S(\mu)$ is fieldlike. Assume that $\alpha_{1}, \ldots, \alpha_{n} \in S(\mu) \backslash\{0,1\}$ with $\sum_{k} \alpha_{k}=1$. Let $[n]={ }_{\text {def }}\{1, \ldots, n\}$ and let $\nu_{n}$ be the measure on $[n]$ with $\nu_{n}(\{k\})=\alpha_{k}$ for $k=1, \ldots, n$. Let $Y=[n]^{\mathbb{Z}}$ with the product topology and let $\nu$ be the product measure on $Y$ with copies of $\nu_{n}$ on each factor. Let $H$ be the shift homeomorphism on $Y$, i.e. $H(y)_{i}=y_{i+1}$ for all $i \in \mathbb{Z}$. Thus, $\nu \in \mathcal{M}_{X}$ and $H \in H_{\nu}(Y)$.

There exists $h \in H_{\mu}(X)$ and an almost one-to-one measure isomorphism $G$ : $X \rightarrow Y$ such that $G$ maps $\mu$ to $\nu$ and $G \circ h=H \circ G$.

Proof. Choose an order on $X$ adapted to $\mu$. Let $F_{\mu}: X \rightarrow I$ be the CDF. For $k=$ $1, \ldots, n$ let $J_{k}$ denote the the $\mu$ interval $\left[\sigma_{k-1}, \sigma_{k}\right]$ with $\sigma_{0}=0$ and $\sigma_{k}={ }_{\text {def }} \sum_{j \leq k} \alpha_{j}$. Thus, the $F_{\mu}$-lifts $\hat{J}_{k}$ for $k=1, \ldots, n$ forms a partition of $X$ with $\mu\left(\hat{J}_{k}\right)=\alpha_{k}$ for 
all $k$. Because $S(\mu)$ is field-like and $\mu$ is adapted to the order, $\tilde{S}\left(\mu_{J_{k}}\right)=S\left(\mu_{J_{k}}\right)=$ $S(\mu)=\tilde{S}(\mu)$ and so there exists a unique order isomorphism $h_{k}:\left(\hat{J}_{k}, \leq\right) \rightarrow(X, \leq)$ which maps $\mu_{\hat{J}_{k}}$ to $\mu$. In the product space $X \times X$ the homeomorphism

$$
h_{k}^{\times} \quad=_{\text {def }} \quad h_{k} \times\left(h_{k}\right)^{-1}: \hat{J}_{k} \times X \rightarrow X \times \hat{J}_{k}
$$

maps $\mu_{\hat{J}_{k}} \times \mu$ to $\mu \times \mu_{\hat{J}_{k}}$. Since the $\mu \times \mu$ measure of each of these pieces is $\alpha_{k}$ we see that $h_{k}^{\times}$is $\mu \times \mu$ measure preserving on each slice. Putting together these pieces we obtain a map $\tilde{h} \in H_{\mu \times \mu}(X \times X)$ which maps the vertical partition to the horizontal partition. Define $\tilde{G}: X \times X \rightarrow Y$ by

$$
\tilde{G}(x, y)_{i}=k \quad \Longleftrightarrow \quad(\tilde{h})^{i}(x, y) \in \hat{J}_{k} \times X \quad \text { for } i \in \mathbb{Z} .
$$

It is easy to see that $\tilde{G}$ maps $\tilde{h}$ to $H$ and $\mu \times \mu$ to $\nu$. Furthermore, if $\tilde{G}\left(x_{1}, y_{1}\right)=$ $\tilde{G}\left(x_{2}, y_{2}\right)$, then $F_{\mu}\left(x_{1}\right)=F_{\mu}\left(x_{2}\right)$ and $F_{\mu}\left(y_{1}\right)=F_{\mu}\left(y_{2}\right)$ because when $X \times X$ is projected to $I \times I$ by $F_{\mu} \times F_{\mu}$, the map $\tilde{h}$ becomes the usual Baker's Transformation associated with $\alpha_{1}, \ldots, \alpha_{n}$. Hence, $\tilde{G}$ is an almost one-to-one surjection and a measure isomorphism from $\mu \times \mu$ to $\nu$.

Because $S(\mu)$ is field-like, Theorem 3.6 implies there exists a homeomorphism $q: X \rightarrow X \times X$ which maps $\mu$ to $\mu \times \mu$. We let $h=q^{-1} \circ \tilde{h} \circ q$ and $G=\tilde{G} \circ q$.

If on a Cantor space $X$ we are given a measure $\mu \in \mathcal{M}_{X}$, a nonempty clopen subset $V$ of $X$ and a positive integer $n$, then a partition $\mathcal{A}$ of $V$ is called a $1 / n$ partition of $V$ when

$$
A \in \mathcal{A} \quad \Longrightarrow \quad \mu(A)=\mu(V) / n .
$$

Clearly a $1 / n$ partition has cardinality $n$.

Recall that $(\Xi, g)$ denotes the universal adding machine, i.e. the Cantor group with $D_{\Xi}$ the entire set of positive integers, i.e. the Cantor group with clopen subgroups of every positive index. By (2.50) and Theorem 2.16, the Haar measure $\mu_{\Xi}$ is characterized up to homeomorphism as the unique good measure with clopen values set

$$
S\left(\mu_{\Xi}\right)=\mathbb{Q} \cap I .
$$

Lemma 4.10. Let $\mu$ be a good measure on a Cantor space $X$.

(a) $\mathbb{Q} \cap I \subset S(\mu)$ iff $X$ admits a $1 / n$ partition for every positive integer $n$.

(b) The following conditions are equivalent:

(1) $S(\mu)$ is $\mathbb{Q}$-like.

(2) For every nonempty clopen subset $V$ of $X S\left(\mu_{V}\right)$ is $\mathbb{Q}$-like.

(3) For every nonempty clopen subset $V$ of $X \mathbb{Q} \cap I \subset S\left(\mu_{V}\right)$.

(4) Every nonempty clopen subset $V$ of $X$ admits a $1 / n$ partition for every positive integer $n$.

(5) With $\mu_{\Xi} \times \mu$ the product measure on $\Xi \times X, S(\mu \Xi \times \mu)=S(\mu)$.

(6) There exists a homeomorphism $q: X \rightarrow \Xi \times X$ such that $q_{*} \mu=\mu_{\Xi} \times \mu$.

Proof. (a) If $X$ admits a $1 / n$ partition, then $n \in \operatorname{Rec}(S(\mu))$. Conversely, if $n \in$ $R(S(\mu))$, then since $S(\mu)$ is group-like, $k / n \in S(\mu)$ for $k=0,1, \ldots, n$. Choose an order on $X$ adapted to $\mu$. As in the proof of Theorem 4.9, $J_{k}=[(k-1) / n, k / n]$ is a $\mu$ interval for $k=1, \ldots, n$ and $\left\{\hat{J}_{k}: k=1, \ldots, n\right\}$ is a $1 / n$ partition of $X$. Hence, $X$ admits such partitions for all $n$ iff $\mathbb{Z}^{*}=\operatorname{Rec}(S(\mu))$ and so by (2.12) iff $\mathbb{Q} \cap I \subset S(\mu)$. 
(b) (1) $\Rightarrow(6)$ : Theorem 3.6.

(6) $\Rightarrow(5)$ : Proposition 1.5.

$(5) \Rightarrow(1)$ : Let $\alpha \in \mathbb{Q}^{*} \cap I$ and $\beta \in S(\mu)$. There exist clopens $U \subset \Xi$ and $V \subset X$ such that $\mu_{\Xi}(U)=\alpha$ and $\mu(V)=\beta$. By assumption (5) $\alpha \beta=\mu_{\Xi} \times \mu(U \times V) \in S(\mu)$. It follows that the group $S(\mu)+\mathbb{Z}$ is closed under multiplication by elements of $\mathbb{Q}$, i.e. $S(\mu)$ is $\mathbb{Q}$-like.

$(1) \Rightarrow(2)$ : Apply (3.3) and Lemma 2.1(d).

(2) $\Rightarrow(3)$ : Obvious.

(3) $\Rightarrow(1)$ : Let $\alpha \in \mathbb{Q}^{*} \cap I$ and $\beta=\mu(V)$ with $V$ clopen in $X$. By assumption (3), $\alpha \in S\left(\mu_{V}\right)$ and so $\alpha \beta \in S(\mu)$.

(3) $\Leftrightarrow(4)$ : Apply part (a) to each $V$.

For a partition $\mathcal{A}$ on a space $X$ the associated equivalence relation $\equiv_{\mathcal{A}}$ is defined to be $\bigcup\{A \times A: A \in \mathcal{A}\}$. If $f_{1}, f_{2}: Y \rightarrow X$ are functions, then we write $f_{1} \equiv_{\mathcal{A}} f_{2}$ if $f_{1}(y) \equiv_{\mathcal{A}} f_{2}(y)$ for all $y \in Y$, i.e. for every $y \in Y$ there exists $A \in \mathcal{A}$ such that $\left\{f_{1}(y), f_{2}(y)\right\} \subset A$. If $\mathcal{A}$ satisfies $\operatorname{mesh}(\mathcal{A}) \leq \epsilon$, then $f_{1} \equiv_{\mathcal{A}} f_{2}$ implies $d\left(f_{1}(y), f_{2}(y)\right) \leq \epsilon$ for all $y \in Y$, i.e. $f_{1}$ and $f_{2}$ are uniformly $\epsilon$ close. On the other hand, if $\epsilon>0$ is the minimum distance between two points in different elements of $\mathcal{A}$, then $d\left(f_{1}(y), f_{2}(y)\right)<\epsilon$ for all $y \in Y$ implies $f_{1} \equiv_{\mathcal{A}} f_{2}$.

If on a Cantor space $X$ we are given a measure $\mu \in \mathcal{M}_{X}$, a nonempty clopen subset $V$ of $X$ and a numbered partition $\left\{A_{1}, \ldots, A_{n}\right\}$ of $V$, then $h \in H_{\mu}(X)$ is said to be cyclic on $V$ of period $n$ with respect to $\left\{A_{1}, \ldots, A_{n}\right\}$ if

$$
\begin{array}{cc}
h^{n}(x)=x & \text { for all } \quad x \in V \\
\text { and } & h\left(A_{i}\right)=A_{i+1}
\end{array}
$$

for $i=1, \ldots, n$ with addition $\bmod n$. Since $h$ is measure preserving, $\left\{A_{1}, \ldots, A_{n}\right\}$ must be a $1 / n$ partition of $V$.

A homeomorphism $h \in H_{\mu}(X)$ is said to be cyclic on $V$ of period $n$ if such a partition exists. It is called a cyclic map of period $n$ if it is a cyclic map of period $n$ on $V=X$ and it is called a cyclic map if it is a cyclic map of period $n$ for some, necessarily unique, positive integer $n$.

If $\mu$ is good on $X$ and $\left\{A_{1}, \ldots, A_{n}\right\}$ is an arbitrary numbered $1 / n$ partition of $V$, then there exist for $i=1, \ldots, n-1$ homeomorphisms $h_{i}: A_{i} \rightarrow A_{i+1}$ mapping $\mu_{A_{i}}$ to $\mu_{A_{i+1}}$. Define

$$
h_{n}=\left(h_{n-1} \circ \ldots \circ h_{1}\right)^{-1}: A_{n} \rightarrow A_{1}
$$

and extend by any measure preserving automorphism of $X \backslash V$, e.g. $1_{X \backslash V}$, to get $h \in H_{\mu}(X)$ which is cyclic on $V$ with respect to $\left\{A_{1}, \ldots, A_{n}\right\}$.

Lemma 4.11. Let $X$ be Cantor space and $\mu \in \mathcal{M}_{X}$. Assume that $h \in H_{\mu}(X), V$ is a nonempty clopen subset of $X$ and $\left\{A_{1}, \ldots, A_{n}\right\}$ is a numbered partition of $V$ such that $h\left(A_{i}\right) \subset A_{i+1}$ for $i=1, \ldots, n$ with addition mod $n$.

(a) $h\left(A_{i}\right)=A_{i+1}$ for $i=1, \ldots, n$ with addition $\bmod n$. If for all $x \in A_{n}, h^{n}(x)=$ $x$, then $h$ is cyclic on $V$ of period $n$ with respect to $\left\{A_{1}, \ldots, A_{n}\right\}$.

(b) Assume that $\mu$ is a good measure on $X$ with $S(\mu) \mathbb{Q}$-like and that $h$ is cyclic on $V$ of period $n$ with respect to $\left\{A_{1}, \ldots, A_{n}\right\}$. For any positive integer $k$ and any partition $\mathcal{B}$ on $X$, there exists a numbered partition $\left\{\tilde{A}_{1}, \ldots . \tilde{A}_{k n}\right\}$ of $V$ and $\tilde{h} \in H_{\mu}(X)$ such that

(1) For $j=1, \ldots, k n$ and $i=1, \ldots, n$, if $i \equiv j \bmod n$, then $\tilde{A}_{j} \subset A_{i}$. 
(2) $\tilde{h}\left(\tilde{A}_{j}\right) \subset \tilde{A}_{j+1}$ for $j=1, \ldots, k n$ with addition $\bmod k n$.

(3) $\tilde{h}^{k n}(x)=x$ for all $x \in V$.

(4) $\tilde{h}(x)=h(x)$ for all $x \in X \backslash A_{n}$.

(5) $\tilde{h}(x) \equiv_{\mathcal{B}} h(x)$ for all $x \in A_{n}$.

In particular, $\tilde{h}$ is cyclic on $V$ of period $k n$ with respect to the refinement $\left\{\tilde{A}_{1}, \ldots, \tilde{A}_{k n}\right\}$ of $\left\{A_{1}, \ldots, A_{n}\right\}$ and $\tilde{h} \equiv_{\mathcal{B}} h$.

Proof. (a) Since $h \in H_{\mu}, \mu\left(A_{i}\right)=\mu\left(h\left(A_{i}\right)\right) \leq \mu\left(A_{i+1}\right)$ for $i=1, \ldots, n$ with addition $\bmod n$. Since these inequalities cycle they are equations. Hence the clopen sets $A_{i+1} \backslash h\left(A_{i}\right)$ have measure zero for $i=1, \ldots, n-1$. Since $\mu$ is full these sets are empty.

If $h^{n}=1_{X}$ on $A_{n}$ and $y \in V$, then $y \in A_{n-i}$ for some $i=0, \ldots, n$ and so $x=h^{i}(y) \in A_{n}$. Hence, $h^{n+i}(y)=h^{n}(x)=x=h^{i}(y)$. Applying $h^{-i}$ we see that $h^{n}(y)=y$.

(b) Because $S(\mu) \mathbb{Q}$-like, on every nonempty clopen of the form $B \cap A_{n}$ with $B \in \mathcal{B}$ we can choose a measure preserving map which is cyclic of period $k$ on $B \cap A_{n}$. Assemble these to obtain a measure preserving map $g$ on $A_{n}$ and extend $g$ by the identity on $X \backslash A_{n}$. Thus, $g(x)=x$ for $x \in X \backslash A_{n}$ and $g(x) \equiv_{\mathcal{B}} x$ for all $x \in A_{n}$. On $A_{n} g$ is cyclic of period $k$ with respect to some numbered partition $C_{1}, \ldots, C_{k}$ of $A_{n}$. Let $\tilde{h}=g \circ h$. Clearly, (4) and (5) hold. For $x \in A_{n}$ we have

$$
\tilde{h}^{i}(x)=h^{i}(x) \quad \text { for } 0 \leq i<n \quad \text { and } \quad \tilde{h}^{n}(x)=g(x) .
$$

Define the numbered partition on $V$ by

$$
\tilde{A}_{i+(r-1) n} \quad=_{\text {def }} \quad h^{i-n}\left(C_{r}\right) \quad \text { for } i=1, \ldots, n \text { and } r=1, \ldots, k .
$$

Obviously (1) holds, and from (4.25), (2) and (3) hold as well.

Now we consider a measure $\mu$ on a Cantor space $X$ with $S(\mu) \mathbb{Q}$-like.

Given any partition $\mathcal{A}$ on $X$ the measure $\mu$ induces the positive distribution $\mu_{\mathcal{A}}$ on $\mathcal{A}$ by

$$
\mu_{\mathcal{A}}(A)=\mu(A) \quad \text { for } A \in \mathcal{A} .
$$

If $f \in H_{\mu}(X)$, then $f$ and $\mu$ induce a distribution $P_{\mathcal{A}}[f]$ on the product set $\mathcal{A} \times \mathcal{A}$ by

$$
P_{\mathcal{A}}[f]\left(A_{1}, A_{2}\right)=\mu\left(A_{1} \cap f^{-1}\left(A_{2}\right)\right)=\mu\left(f\left(A_{1}\right) \cap A_{2}\right) \quad \text { for } A_{1}, A_{2} \in \mathcal{A} .
$$

Clearly,

$$
\sum_{B \in \mathcal{A}} P_{\mathcal{A}}[f](A, B)=\sum_{B \in \mathcal{A}} P_{\mathcal{A}}[f](B, A)=\mu(A) \quad \text { for } A \in \mathcal{A} .
$$

The associated relation $R_{\mathcal{A}}[f]$ on $\mathcal{A}$ is the subset of $\mathcal{A} \times \mathcal{A}$ given by

$$
R_{\mathcal{A}}[f]=\left\{\left(A_{1}, A_{2}\right): A_{1} \cap f^{-1}\left(A_{2}\right) \neq \emptyset\right\}=\left\{\left(A_{1}, A_{2}\right): P_{\mathcal{A}}[f]\left(A_{1}, A_{2}\right)>0\right\} .
$$

Lemma 4.12. The transitive relation $\mathcal{O} R_{\mathcal{A}}[f]=d_{\text {def }} \bigcup_{k=1}^{\infty}\left(R_{\mathcal{A}}[f]\right)^{k}$ is an equivalence relation.

Proof. From (4.29) it follows that $R=R_{\mathcal{A}}[f]$ is a surjective relation. That is, for every $A \in \mathcal{A}$ there exist $B_{1}, B_{2}$ such that $P_{\mathcal{A}}[f]\left(A, B_{1}\right)$ and $P_{\mathcal{A}}[f]\left(B_{2}, A\right)$ are positive. We prove that $\mathcal{O} R$ is an equivalence relation on $\mathcal{A}$ by induction on the cardinality $n$ of $\mathcal{A}$.

If $n=1$, then $\mathcal{A}=\{X\}$ and $R=\{(X, X)\}$ is the trivial equivalence relation. 
On the cyclic set of $\mathcal{O} R$,

$$
|\mathcal{O} R| \quad=_{\text {def }} \quad\{A \in \mathcal{A}:(A, A) \in \mathcal{O} R\},
$$

$\mathcal{O} R \cap(\mathcal{O} R)^{-1}$ is an equivalence relation and its equivalence classes, the $R$ basic sets, are partially ordered by $\mathcal{O} R$. Choose $\mathcal{B}$ a terminal basic set. That is, $\mathcal{B} \times \mathcal{B} \subset \mathcal{O} R$ and

$$
B \in \mathcal{B} \text { and }(B, A) \in \mathcal{O} R \quad \Longrightarrow \quad A \in \mathcal{B} \text {. }
$$

From (4.29) and (4.32) we have

$$
\begin{array}{r}
\sum_{B \in \mathcal{B}} \sum_{A \in \mathcal{A}} P_{\mathcal{A}}[f](A, B)=\sum_{B \in \mathcal{B}} \mu(B) \\
=\sum_{B \in \mathcal{B}} \sum_{A \in \mathcal{A}} P_{\mathcal{A}}[f](B, A)=\sum_{A, B \in \mathcal{B}} P_{\mathcal{A}}[f](B, A),
\end{array}
$$

since $B \in \mathcal{B}$ and $A \notin \mathcal{B}$ implies $P_{\mathcal{A}}[f](B, A)=0$. It follows that $P_{\mathcal{A}}[f](A, B)=0$ if $B \in \mathcal{B}$ and $A \notin \mathcal{B}$.

Now if $\mathcal{A}=\mathcal{B}$, then $\mathcal{O} R=\mathcal{B} \times \mathcal{B}$ is an equivalence relation. If $\tilde{\mathcal{A}}=\mathcal{A} \backslash \mathcal{B}$ is nonempty, then it is a partition of cardinality less than $n$ on a nonempty clopen subset $V$ of $X$. Furthermore, $P_{\mathcal{A}}[f](B, A)=P_{\mathcal{A}}[f](A, B)=0$ if $A \in \tilde{\mathcal{A}}$ and $B \in \mathcal{B}$. Hence, $f(V)=V$ and $f \in H_{\mu_{V}}(V)$. Applying the inductive hypothesis we have that $\mathcal{O} R_{\tilde{\mathcal{A}}}[f]$ is an equivalence relation and so

$$
\mathcal{O} R_{\mathcal{A}}[f]=\mathcal{O} R_{\tilde{\mathcal{A}}}[f] \cup(\mathcal{B} \times \mathcal{B})
$$

is an equivalence relation as well.

Theorem 4.13. If $\mu$ is a good measure on a Cantor space $X$ such that $S(\mu)$ is $\mathbb{Q}$-like, then the set of cyclic functions is dense in $H_{\mu}(X)$.

Proof. Given a partition $\mathcal{A}$, let $P$ denote the $\mathcal{A} \times \mathcal{A}$ matrix $P_{\mathcal{A}}[f]$. We use induction on number $N$ of nonzero entries in $P$ to prove that there exists a cyclic function $g$ in $H_{\mu}$ such that $g \equiv_{\mathcal{A}} f$.

If $N=1$, then $\mathcal{A}=\{X\}$ and $g=1_{X}$ is a cyclic function with $g \equiv_{\mathcal{A}} f$.

For the inductive step we first construct a cycle on a nonempty clopen part of the space.

Case i: Some diagonal entry $P\left(A_{1}, A_{1}\right)$ is positive.

Define $Z_{1}=A_{1} \cap f^{-1}\left(A_{1}\right)$. By (4.28) $\mu\left(Z_{1}\right)=P\left(A_{1}, A_{1}\right)$. Since $f\left(Z_{1}\right)=$ $f\left(A_{1}\right) \cap A_{1}$ and $f \in H_{\mu}$ we have $\mu\left(A_{1} \backslash f\left(Z_{1}\right)\right)=\mu\left(A_{1} \backslash Z_{1}\right)$. Because $\mu$ is good we can choose $h \in H_{\mu}$ such that

$$
\begin{aligned}
& h\left(A_{1} \backslash f\left(Z_{1}\right)\right)=A_{1} \backslash Z_{1}, \\
& h=f^{-1} \quad \text { on } f\left(Z_{1}\right) \text {, } \\
& h=1_{X} \quad \text { on } \quad X \backslash A_{1} .
\end{aligned}
$$

Clearly, $h \equiv_{\mathcal{A}} 1_{X}$ and so with $f_{1}=_{\operatorname{def}} h \circ f f_{1} \equiv_{\mathcal{A}} f$. Let $Z=Z_{1}$. On $Z f_{1}$ restricts to the identity. That is, it is cyclic of period $n=1$.

Case ii: With $A_{1} \neq A_{2} \in \mathcal{A}, p={ }_{\text {def }} P\left(A_{1}, A_{2}\right)$ is the smallest positive entry of $P$.

By Lemma 4.12 we can construct a chain $\left\{A_{1}, A_{2}, \ldots, A_{n}\right\}$ in $\mathcal{A}$ so that with $A_{n+1}={ }_{\text {def }} A_{1}$ we have $P\left(A_{k}, A_{k+1}\right)>0$ for $k=1,2, \ldots, n$. By assumption, $P\left(A_{k}, A_{k+1}\right) \geq p$ for $k=1,2, \ldots, n$. Suppose $n \geq 2$ is the minimal length of a chain 
satisfying these properties. If for some $2 \leq j<k \leq n, A_{j}=A_{k}$, then we could remove $A_{j+1}, \ldots, A_{k}$ to get a shorter list. Similarly, if for some $2<k \leq n, A_{k}=A_{1}$ we could remove $A_{k}, \ldots, A_{n}$. Thus, the chain of minimal length has distinct members in $\mathcal{A}$.

Define $Z_{1}=A_{1} \cap f^{-1}\left(A_{2}\right)$ so that $f\left(Z_{1}\right) \subset A_{2}$ and

$$
\mu\left(f\left(Z_{1}\right)\right)=p \leq \mu\left(A_{2} \cap f^{-1}\left(A_{3}\right)\right) .
$$

By Proposition 2.11 applied to the good measure $\mu_{A_{2}}$ there exists $h_{2} \in H_{\mu_{A_{2}}}\left(A_{2}\right)$ such that

$$
Z_{2} \quad=_{\text {def }} \quad h_{2}\left(f\left(Z_{1}\right)\right) \quad \subset \quad A_{2} \cap f^{-1}\left(A_{3}\right),
$$

and so $\mu\left(Z_{2}\right)=p$.

Inductively, for $k=3, \ldots, n$ we construct $h_{k} \in H_{\mu_{A_{k}}}\left(A_{k}\right)$ and $Z_{k} \subset A_{k} \cap$ $f^{-1}\left(A_{k+1}\right)$ with $\mu\left(Z_{k}\right)=p$ such that

$$
Z_{k} \quad=_{\text {def }} \quad h_{k}\left(f\left(Z_{k-1}\right)\right) \quad \subset \quad A_{k} \cap f^{-1}\left(A_{k+1}\right) .
$$

Since $A_{n+1}=A_{1}$ (4.38) implies that $Z_{1}$ and $f\left(Z_{n}\right)$ are clopen subsets of $A_{1}$ with the same measure. We can choose $h \in H_{\mu}$ such that

$$
\begin{aligned}
& h\left(A_{1} \backslash f\left(Z_{n}\right)\right)=A_{1} \backslash Z_{1}, \\
& h=\left(f \circ h_{n} \circ f \circ h_{n-1} \circ \ldots \circ h_{2} \circ f\right)^{-1} \quad \text { on } f\left(Z_{n}\right) \text {, } \\
& h=h_{k} \quad \text { on } \quad A_{k} \quad \text { for } k=2, \ldots, n \text {, } \\
& h=1_{X} \quad \text { on } \quad X \backslash\left(\bigcup_{j=1}^{n} A_{j}\right) .
\end{aligned}
$$

Clearly, $h \equiv_{\mathcal{A}} 1_{X}$ and so with $f_{1}=_{\operatorname{def}} h \circ f, f_{1} \equiv_{\mathcal{A}} f$. With respect to the numbered partition $\left\{Z_{1}, \ldots, Z_{n}\right\}$ of $Z={ }_{\text {def }} \bigcup_{j=1}^{n} Z_{j}, f_{1}$ is cyclic on $Z$ of period $n$.

So in each case, we have constructed $f_{1} \equiv_{\mathcal{A}} f$ cyclic of period $n$ on the clopen subset $Z$ of $X$.

If $Z=X$ we have constructed the desired cyclic function on $X$. It remains to consider the case when $\tilde{X}=X \backslash Z$ is a nonempty clopen subset with the good measure $\tilde{\mu}=\mu_{\tilde{X}}$ and the partition $\tilde{\mathcal{A}}=\{A \cap \tilde{X}: A \in \mathcal{A}\} \backslash\{\emptyset\}$. Notice that $Z$ is an invariant subset for $f_{1}$ and so letting $\tilde{f}$ denote the restriction of $f_{1}$ to $\tilde{X}$ we have $\tilde{f} \in H_{\tilde{\mu}}(\tilde{X})$. So if we let

$$
\tilde{P}(A, B) \quad=_{\text {def }} \quad \mu\left(A \cap f_{1}^{-1}(B) \cap \tilde{X}\right) / \mu(\tilde{X}),
$$

then $\tilde{P}$ is the matrix $P_{\tilde{\mathcal{A}}}[\tilde{f}]$ extended by 0 for those indices of $A \in \mathcal{A}$ such that $A \cap \tilde{X}=\emptyset$. Since $f_{1} \equiv_{\mathcal{A}} f$ we have for all $A, B \in \mathcal{A}$

$$
A \cap f^{-1}(B)=A \cap f_{1}^{-1}(B) .
$$

Consequently,

$$
\tilde{P}(A, B) \leq P(A, B) / \mu(\tilde{X}) .
$$

Furthermore, since $Z_{1}$ is disjoint from $\tilde{X}$,

$$
\begin{aligned}
& \tilde{P}\left(A_{1}, A_{1}\right)=0 \quad \text { in Case i, } \\
& \tilde{P}\left(A_{1}, A_{2}\right)=0 \quad \text { in Case ii. }
\end{aligned}
$$

Thus, $P_{\tilde{\mathcal{A}}}[\tilde{f}]$ has fewer positive entries than does $P_{\mathcal{A}}[f]$. It follows from the induction hypothesis that there exists $\tilde{f}_{1} \in H_{\tilde{\mu}}(\tilde{X})$ such that $\tilde{f}_{1} \equiv_{\tilde{\mathcal{A}}} \tilde{f}$ and $\tilde{f}_{1}$ is 
cyclic on $\tilde{X}$ with period $\tilde{n}$. Define $f_{2} \in H_{\mu}(X)$ to be $f_{1}$ on $Z$ and $\tilde{f}_{1}$ on the complement $\tilde{X}=X \backslash Z$. Thus, $f_{2} \equiv_{\mathcal{A}} f$ and $f_{2}$ is cyclic of period $n$ on $Z$ and of period $\tilde{n}$ on $\tilde{X}$. Apply Lemma 4.11(a) to change $f_{2}$ on each of the two pieces $Z$ and $\tilde{X}$ of $X$ to get $f_{3} \equiv_{\mathcal{A}} f_{2} \equiv_{\mathcal{A}} f$ which is cyclic of period $n \cdot \tilde{n}$ on each piece.

Recall that a partition $\mathcal{A}_{1}$ refines $\mathcal{A}_{2}$, written $\mathcal{A}_{1} \rightarrow \mathcal{A}_{2}$, if each element of $\mathcal{A}_{1}$ is contained in a member of $\mathcal{A}_{2}$ and we define the surjection $\pi_{\mathcal{A}_{1}}^{\mathcal{A}_{2}}: \mathcal{A}_{1} \rightarrow \mathcal{A}_{2}$ by

$$
A \subset \pi_{\mathcal{A}_{1}}^{\mathcal{A}_{2}}(A) \quad \text { for } A \in \mathcal{A}_{1} .
$$

If $\mathcal{A}_{1}$ and $\mathcal{A}_{2}$ are partitions of $X$, then the least common refinement is

$$
\mathcal{A}_{1} \wedge \mathcal{A}_{2} \quad=_{\text {def }} \quad\left\{A_{1} \cap A_{2}: A_{1} \in \mathcal{A}_{1} \text { and } A_{2} \in \mathcal{A}_{2}\right\} \backslash\{\emptyset\} .
$$

If $f$ is an automorphism of $X$, then a partition $\mathcal{A}$ is called invariant if

$$
A \in \mathcal{A} \quad \Longrightarrow \quad f(A) \in \mathcal{A} \text {. }
$$

This says exactly that the relation $R_{\mathcal{A}}[f]$ is a function on $\mathcal{A}$ in which case we will denote it by $f_{\mathcal{A}}$. Since the relation $R_{\mathcal{A}}[f]$ is always surjective and $\mathcal{A}$ is finite, $f_{\mathcal{A}}$ is a permutation of the invariant partition $\mathcal{A}$. The partition is called fixed if

$$
A \in \mathcal{A} \quad \Longrightarrow \quad f(A)=A \text {. }
$$

That is, $\mathcal{A}$ is invariant and $f_{\mathcal{A}}=1_{\mathcal{A}}$. At the opposite extreme, the partition is called cyclic if it is invariant and the permutation $f_{\mathcal{A}}$ consists of a single cycle on $\mathcal{A}$.

If $\mathcal{A}_{1} \rightarrow \mathcal{A}_{2}$ and $\mathcal{A}_{1}$ is invariant, then $\mathcal{A}_{2}$ invariant and

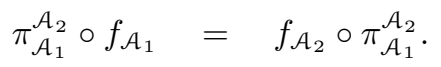

If $\mathcal{A}_{1}$ is fixed (or cyclic), then $\mathcal{A}_{2}$ is fixed (resp. cyclic).

If $\mathcal{A}_{1}$ and $\mathcal{A}_{2}$ are invariant (or fixed) partitions, then $\mathcal{A}_{1} \wedge \mathcal{A}_{2}$ is invariant (resp. fixed). However, $\mathcal{A}_{1} \wedge \mathcal{A}_{2}$ is not ncessarily cyclic when the two factors are.

If $\mathcal{A}$ is an invariant partition, then we can concatenate the elements of the separate cycles of the permutation $f_{\mathcal{A}}$ to obtain the fixed partition associated with $\mathcal{A}$ denoted $[\mathcal{A}]$. Thus, $[\mathcal{A}]$ is the finest fixed partition of which $\mathcal{A}$ is a refinement. $\mathcal{A}$ is a cyclic partition iff it is invariant and $[\mathcal{A}]$ is the trivial partition $\{X\}$.

Definition 4.14. Let $X$ be a Cantor space equipped with a metric $d$ and a measure $\mu \in \mathcal{M}_{X}$. Let $\mathcal{A}=\left\{A_{1}, \ldots, A_{n}\right\}$ be a numbered partition of $X, \epsilon$ be a positive rational, $N$ be a positive integer and $\alpha:[n] \rightarrow I \backslash\{0,1\}$ be a function which satisfies

$$
\alpha_{i} \in S\left(\mu_{A_{i}}\right) \quad \text { for } \quad i \in[n]=\{1,2, \ldots, n\} .
$$

$f \in H_{\mu}(X)$ is in the subset $G(\mathcal{A}, \alpha, \epsilon, N)$ if either $\mathcal{A}$ is not a fixed partition for $f$ or there exist positive integers $k, M$ and a partition $\mathcal{W}$ indexed by $[n] \times[2] \times[M] \times$ $[k N]$ so that for $i j s r \in[n] \times[2] \times[M] \times[k N]$

(i) $W_{i j s r} \subset A_{i}$.

(ii) $\mu\left(W_{i 1 s r}\right)=\alpha_{i} \mu\left(W_{i 1 s r} \cup W_{i 2 s r}\right)$.

(iii) $d\left(W_{i j s r}\right)<\epsilon$.

(iv) $f\left(W_{i j s r}\right)=W_{i j s(r+1)} \quad$ with addition $\bmod k N$.

Lemma 4.15. Each $G(\mathcal{A}, \alpha, \epsilon, N)$ is an open subset of $H_{\mu}(X)$. If $\mu$ is a good measure on $X$ with $S(\mu) \mathbb{Q}$-like, then each $G(\mathcal{A}, \alpha, \epsilon, N)$ is dense in $H_{\mu}(X)$. 
Proof. If $f \equiv_{\mathcal{A}} f_{1}$, then the relations $R_{\mathcal{A}}[f]$ and $R_{\mathcal{A}}\left[f_{1}\right]$ agree. So $\mathcal{A}$ is invariant, fixed or cyclic for $f_{1}$ if it satisfies the corresponding property for $f$. Thus, if $\mathcal{A}$ is not fixed for $f$, then it is not fixed for $f_{1}$. On the other hand, for the partition $\mathcal{W}$ conditions (i), (ii) and (iii) do not depend on $f$ while if (iv) holds for $f$, then it holds for any $f_{1}$ such that $f_{1} \equiv \mathcal{W} f$. Thus, $G=G(\mathcal{A}, \alpha, \epsilon, N)$ is an open subset of $H_{\mu}$.

Now assume that $\mu$ is good on $X$ with $S(\mu) \mathbb{Q}$-like, $\mathcal{B}$ is an arbitrary partition of $X$ and that $f \in H_{\mu}(X)$. We will construct $g \equiv_{\mathcal{B}} f$ with $g \in G$. If $\mathcal{A}$ is not fixed for $f$, then $f \in G$ and we can let $g=f$. Assume now that $\mathcal{A}$ is fixed partition for $f$. Furthermore, by replacing $\mathcal{B}$ by $\mathcal{B} \wedge \mathcal{A}$ if necessary, we can assume that

$$
\mathcal{B} \rightarrow \mathcal{A} \text {. }
$$

By Theorem 4.13 and Lemma 4.11(b) there exists a positive integer $k$ and a map $g \in H_{\mu}$ with $g \equiv_{\mathcal{B}} f$ such that $g$ is a cyclic map of period $k N$. That is, there is a partition $\left\{W_{r}: r \in[k N]\right\}$ with $W_{k N+1}=W_{1}$ such that $g\left(W_{r}\right)=W_{r+1}$ for all $r$ and $g^{k N}=1_{X}$.

Since $\mathcal{B}$ refines $\mathcal{A}, g \equiv_{\mathcal{A}} f$ and so $\mathcal{A}$ is a fixed partition for $g$. So if $W_{i r}=A_{i} \cap W_{r}$, then $g\left(W_{i r}\right)=W_{i(r+1)}$. Hence, the $g$ orbit of any $x \in A_{i}$ moves cyclically through the $W_{i r}$ 's and so no $W_{i r}$ is empty.

Choose $\delta>0$ an $\epsilon$ modulus of uniform continuity for $1_{X}, g, \ldots, g^{k N}$. Because $S(\mu)$ is $\mathbb{Q}$-like, we can choose a positive integer $M$ large enough that each $W_{i 1}$ can be partitioned into exactly $M$ clopen pieces of equal measure and of diameter less than $\delta$. These pieces are labeled $W_{i s 1}$ for $s \in[M]$. Define $W_{i s r}=g^{r-1}\left(W_{i s 1}\right)$ for $r \in[k N]$. Thus,

$$
d\left(W_{i s r}\right)<\epsilon \quad \text { and } \quad \mu\left(W_{i s r}\right)=\mu\left(A_{i}\right) / k N M .
$$

By assumption on the map $\alpha$, each $\mu\left(A_{i}\right) \alpha_{i} \in S(\mu)$. Since $S(\mu)$ is $\mathbb{Q}$-like, $\mu\left(A_{i}\right) \alpha_{i} / k N M \in S(\mu)$. So by the Subset Condition we can choose a clopen $W_{i 1 s 1} \subset W_{i s 1}$ with this measure and let $W_{i 2 s 1}=W_{i s 1} \backslash W_{i 1 s 1}$. Since $\alpha_{i} \neq 0,1$ these sets are nonempty. Finally, define $W_{i j s r}=g^{r-1}\left(W_{i j s 1}\right)$ for $r \in[k N]$ so that

$$
\mu\left(W_{i 1 s r}\right)=\alpha_{i} \mu\left(W_{i s r}\right) .
$$

Notice that $g^{k N}=1_{X}$ implies that $g\left(W_{i j s(k N)}\right)=W_{i j s 1}$ as required.

Define the subset

$$
H_{\mu}^{*} \quad=_{\text {def }} \bigcap\{G(\mathcal{A}, \alpha, \epsilon, N)\}
$$

with $\mathcal{A}, \alpha, \epsilon, N$ varying as in Definition 4.14 .

Lemma 4.16. If $\mu$ is a good measure on $X$ with $S(\mu) \mathbb{Q}$-like, then $H_{\mu}^{*}$ is a dense, $G_{\delta}$, conjugacy invariant subset of the Polish group $H_{\mu}(X)$.

Proof. Because the index set in Definition 4.14 is countable, Lemma 4.15 and the Baire Category Theorem imply that $H_{\mu}^{*}$ is a dense, $G_{\delta}$ subset of $H_{\mu}$.

Now let $f \in H_{\mu}^{*}, h \in H_{\mu}$ and $\mathcal{A}, \alpha, \epsilon, N$ be in the index set of Definition 4.14. Let $\delta>0$ be a positive rational modulus of uniform continuity for $h$ and let

$$
h^{-1} \mathcal{A} \quad=_{\text {def }} \quad\left\{h^{-1}(A): A \in \mathcal{A}\right\}
$$

be the pulled back partition. Since $\mu\left(h^{-1}(A)\right)=\mu(A)$ the function $\alpha$ satisfies condition (4.49) for $h^{-1} \mathcal{A}$. 
By assumption $f \in G\left(h^{-1} \mathcal{A}, \alpha, \delta, N\right)$. If $\mathcal{A}$ is fixed for $h f h^{-1}$, then $h^{-1} \mathcal{A}$ is fixed for $f$. Then if $\mathcal{W}$ satisfies (i)-(iv) of Definition 4.14 for $f$ with $\epsilon$ replaced by $\delta$, then $\left\{h\left(W_{i j s r}\right\}\right.$ satisfies (i)-(iv) for $h f h^{-1}$. Thus, the conjugate $h f h^{-1} \in H_{\mu}^{*}$.

Now we show that when $S(\mu)$ is $\mathbb{Q}$-like, the dense $G_{\delta}$ subset $H_{\mu}^{*}$ is a single conjugacy class in the group $H_{\mu}(X)$. Thus, the group $H_{\mu}(X)$ satisfies the Strong Rohlin Property.

Theorem 4.17. Let $(\Xi, g)$ be the universal adding machine Cantor group with the Haar measure $\mu_{\Xi}$ and let $h \in H_{\mu \Xi}(\Xi)$ be translation by the generator $g$. Let $\mu$ be a good measure on $X$ with $S(\mu) \mathbb{Q}$-like and let $H_{\mu}^{*}$ be the dense, $G_{\delta}$ subset of $H_{\mu}(X)$ defined by equation (4.53). If $f \in H_{\mu}(X)$, then $f \in H_{\mu}^{*}$ iff there exists a homeomorphism $q: X \rightarrow X \times \Xi$ such that

$$
q_{*} \mu=\mu \times \mu_{\Xi}
$$

and

$$
q \circ f=\left(1_{X} \times h\right) \circ q .
$$

In particular, $H_{\mu}^{*}$ is a single conjugacy class in the group $H_{\mu}$.

Proof. The existence of $q$ satisfying (4.55) and (4.56) says exactly that $f$ on $X$ is conjugate to the fixed map $1_{X} \times h$ on the product $X \times \Xi$. So the set of such $f$ in $H_{\mu}$, if nonempty, consists of a single conjugacy class. If this class contains $H_{\mu}^{*}$, then it equals $H_{\mu}^{*}$ because this set is conjugacy invariant by Lemma 4.16.

It remains to show that for a given $f \in H_{\mu}^{*}$ there exists $q$ satifying (4.55) and (4.56).

The partitions which are fixed by $f$ are directed by refinement. Define $X_{F}$ to be the inverse limit space. So for each fixed partition $\mathcal{A}$ there is a projection map $\pi_{F}^{\mathcal{A}}: X_{F} \rightarrow \mathcal{A}$. For each $x \in X$ the carriers $\pi^{\mathcal{A}}(x)$ defined by (2.30) define a point $\pi^{F}(x) \in X_{F}$. So we obtain $\pi^{F}: X \rightarrow X_{F}$ such that

$$
\pi_{F}^{\mathcal{A}} \circ \pi^{F}=\pi^{\mathcal{A}}: X \rightarrow \mathcal{A} .
$$

Since $f$ induces the identity on each fixed partition it is clear that $\pi^{F}$ maps $f$ to $1_{X_{F}}$.

Define $\mu_{F}$ on $X_{F}$ by

$$
\mu_{F} \quad=_{\text {def }}\left(\pi^{F}\right)_{*} \mu .
$$

If $z \in X_{F}$, then

$$
\left(\pi^{F}\right)^{-1}(z)=\bigcap\left\{\pi_{F}^{\mathcal{A}}(z): \mathcal{A} \text { fixed }\right\} .
$$

By compactness this set is not empty and so $\pi^{F}$ is surjective and $\mu_{F}$ is full.

If $V$ is a clopen subset of $X_{F}$, then there exists a fixed partition $\mathcal{A}=\left\{A_{1}, \ldots, A_{n}\right\}$ numbered so that for some $p \in[n]$

$$
V=\bigcup_{i=1}^{p}\left(\pi_{F}^{\mathcal{A}}\right)^{-1}\left(A_{i}\right)
$$

and so

$$
\left(\pi^{F}\right)^{-1}(V)=\bigcup_{i=1}^{p} A_{i} \quad \text { and } \quad \mu_{F}(V)=\sum_{i=1}^{p} \mu\left(A_{i}\right) .
$$


Now suppose that $\gamma \in S(\mu) \backslash\{0\}$ and $\gamma<\mu_{F}(V)$. Let $q$ be the element of $[p]$ so that

$$
\sum_{i=1}^{q-1} \mu\left(A_{i}\right)<\gamma \leq \sum_{i=1}^{q} \mu\left(A_{i}\right)
$$

and let

$$
\alpha_{q} \quad=_{\text {def }} \quad\left(\gamma-\sum_{i=1}^{q-1} \mu\left(A_{i}\right)\right) / \mu\left(A_{q}\right) .
$$

If $\alpha_{q}=1$, i.e. equality holds on the right in (4.62), then $U=\bigcup_{i=1}^{q}\left(\pi_{F}^{\mathcal{A}}\right)^{-1}\left(A_{i}\right)$ is a clopen subset of $V$ with $\mu_{F}$ measure $\gamma$.

Now assume that $\alpha_{q}<1$ so that $\alpha_{q} \in S\left(\mu_{A_{q}}\right) \backslash\{0,1\}$. Because $f \in H_{\mu}^{*}$ it is in $G(\mathcal{A}, \alpha, 1,1)$ with $\alpha$ any map satisfying (4.49) but with $\alpha_{q}$ given by (4.63). Let $\mathcal{W}$ be a partition which satisfies (i)-(iv) of Definition 4.14 and let

$$
\begin{array}{r}
A_{q}^{-} \quad=_{\text {def }} \quad \bigcup\left\{W_{q 1 s r}: s \in[M] \text { and } r \in[k]\right\}, \\
A_{q}^{+}={ }_{\text {def }} \quad \bigcup\left\{W_{q 1 s r}: s \in[M] \text { and } r \in[k]\right\}=A_{q} \backslash A_{q}^{-} .
\end{array}
$$

By condition (iv), $\tilde{\mathcal{A}}=\left\{A_{1}, \ldots, A_{q-1}, A_{q}^{-}, A_{q}^{+}, A_{q+1}, \ldots, A_{n}\right\}$ is a fixed partition refining $\mathcal{A}$ and by (iii)

$$
\mu\left(A_{q}^{-}\right)=\alpha_{q} \mu\left(A_{q}\right)=\gamma-\sum_{i=1}^{q-1} \mu\left(A_{i}\right) .
$$

It follows that $U=\left(\bigcup_{i=1}^{q-1}\left(\pi_{F}^{\tilde{\mathcal{A}}}\right)^{-1}\left(A_{i}\right)\right) \cup\left(\pi_{F}^{\tilde{\mathcal{A}}}\right)^{-1}\left(A_{q}^{-}\right)$is a clopen subset of $V$ with $\mu_{F}$ measure $\gamma$.

First, this shows that $X_{F}$ has no isolated points and so, as the countable inverse limit of finite sets, it is a Cantor space. Next, since $\gamma$ can be chosen from elements of a dense subset of $\left(0, \mu_{F}(V)\right)$ it follows that the measure $\mu_{F}$ is nonatomic. Thus, $\mu_{F} \in \mathcal{M}_{X_{F}}$. Then by applying the argument to $V=X_{F}$ we see that $S(\mu) \subset S\left(\mu_{F}\right)$. The reverse inclusion follows from Proposition 1.5 and so

$$
S\left(\mu_{F}\right)=S(\mu) .
$$

Finally, the argument for general clopen $V$ is a direct verification of the Subset Condition and so $\mu_{F}$ is a good measure on $X_{F}$.

For the adding machine factor an inductive construction with repeated choices is required because the cyclic partitions are not directed by refinement.

On the finite pointed cyclic group $\left(\mathbb{Z}_{k}, 1\right)$ of integers mod $k$, let $\mu_{k}$ be the Haar measure, i.e. the uniform distribution, and let $h_{k}$ be the 1 translation map.

If $\mathcal{A}$ is an invariant partition for $f$, then a $\mathbb{Z}_{k}$ label for $\mathcal{A}$ is a map $u_{k}: \mathcal{A} \rightarrow \mathbb{Z}_{k}$ such that

$$
u_{k} \circ f_{\mathcal{A}}=h_{k} \circ u_{k} .
$$

That is, $u_{k}$ maps $f_{\mathcal{A}}$ to $h_{k}$. Since $\mu_{k}$ is the only $h_{k}$ invariant measure, (4.27) and (4.67) imply

$$
u_{k *} \mu_{\mathcal{A}}=\mu_{k}
$$


see Proposition 4.4(d). With $[\mathcal{A}]$ the fixed partition associated with $\mathcal{A}$ obtained by concatenating the elements of each cycle of $f_{\mathcal{A}}$ we have

$$
\begin{array}{r}
{\left[u_{k}\right]={ }_{\text {def }} \pi_{\mathcal{A}}^{[\mathcal{A}]} \times u_{k}: \mathcal{A} \rightarrow[\mathcal{A}] \times \mathbb{Z}_{k},} \\
{\left[u_{k}\right] \circ f_{\mathcal{A}}=1_{[\mathcal{A}]} \times h_{k} \circ\left[u_{k}\right],} \\
{\left[u_{k}\right]_{*} \mu_{\mathcal{A}}=\mu_{[\mathcal{A}]} \times \mu_{k}}
\end{array}
$$

because the only $1_{[\mathcal{A}]} \times h_{k}$ invariant measure which projects to $\mu_{[\mathcal{A}]}$ is the product measure.

Of course, an invariant partition $\mathcal{A}$ admits a $\mathbb{Z}_{k}$ label iff every cycle in the permutation $f_{\mathcal{A}}$ has length divisible by $k$. Given the label, $\left[u_{k}\right]$ is surjective and it is bijective iff every $f_{\mathcal{A}}$ cycle has length exactly $k$.

Recall that $f \in H_{\mu}^{*}$. Hence, for every positive rational $\epsilon$ and positive integer $N$ there exists a positive integer $k$ and an invariant partition $\mathcal{W}(\epsilon, k N)$ whose mesh is less than $\epsilon$ and such that every $f_{\mathcal{W}}$ cycle has length $k N$. Hence, there is a $\mathbb{Z}_{k N}$ label $u_{k N}$ on $\mathcal{W}$ such that $\left[u_{k N}\right]$ is a bijection from $\mathcal{W}$ to $[\mathcal{W}] \times \mathbb{Z}_{k N}$. In fact, with the indexing given by (i)-(iv) of Definition 4.14 the $r$ index provides the map $u_{k N}$ and ijs indexes the elements of $[\mathcal{W}]$.

We will need one other construction.

Suppose that $k_{1} \mid k_{2}$. The natural projection $\pi_{k_{2}}^{k_{1}}:\left(\mathbb{Z}_{k_{2}}, 1\right) \rightarrow\left(\mathbb{Z}_{k_{1}}, 1\right)$ maps $h_{k_{2}}$ to $h_{k_{1}}$. On the product group $\mathbb{Z}_{k_{1}} \times \mathbb{Z}_{k_{2}}$ the product map $h_{k_{1}} \times h_{k_{2}}$ is translation by the element $(1,1)$ and every orbit is periodic with period $k_{2}$.

These groups are in fact rings and $\pi_{k_{2}}^{k_{1}}$ is a ring homomorphism. So we can regard the product $\mathbb{Z}_{k_{1}} \times \mathbb{Z}_{k_{2}}$ as a module over $\mathbb{Z}_{k_{2}}$ and so can multiply by elements of $\mathbb{Z}_{k_{2}}$.

An element $(i, j) \in \mathbb{Z}_{k_{1}} \times \mathbb{Z}_{k_{2}}$ can be written uniquely as

$$
(i, j)=(k, 0)+j(1,1) \quad \text { with } \quad k=i-\pi_{k_{2}}^{k_{1}}(j) .
$$

Now choose a set map $\tilde{v}: \mathbb{Z}_{k_{1}} \rightarrow \mathbb{Z}_{k_{2}}$ which is a right inverse for the surjection $\pi_{k_{2}}^{k_{1}}$. That is, $\pi_{k_{2}}^{k_{1}} \circ \tilde{v}$ is the identity on $\mathbb{Z}_{k_{1}}$. Now extend $\tilde{v}$ to map $\mathbb{Z}_{k_{1}} \times \mathbb{Z}_{k_{2}}$ onto $\mathbb{Z}_{k_{1}}$ so that

$$
(i, j)=(k, 0)+j(1,1) \quad \Longrightarrow \quad v(i, j)=\tilde{v}(k)+j .
$$

That is, from (4.70)

$$
v(i, j)=\tilde{v}\left(i-\pi_{k_{2}}^{k_{1}}(j)\right)+j .
$$

Since $\pi_{k_{2}}^{k_{1}}$ is a homomorphism, it follows that

$$
\pi_{k_{2}}^{k_{1}} \circ v(i, j)=i .
$$

Furthermore,

$$
\begin{array}{r}
v\left((i, j)+j_{1}(1,1)\right)=v\left(i+\pi_{k_{2}}^{k_{1}}\left(j_{1}\right), j+j_{1}\right) \\
\quad=\tilde{v}\left(i+\pi_{k_{2}}^{k_{1}}\left(j_{1}\right)-\pi_{k_{2}}^{k_{1}}\left(j+j_{1}\right)\right)+j+j_{1} \\
\quad=\tilde{v}\left(i-\pi_{k_{2}}^{k_{1}}(j)\right)+j+j_{1}=v(i, j)+j_{1} .
\end{array}
$$

That is, $v$ maps $h_{k_{1}} \times h_{k_{2}}$ to $h_{k_{2}}$.

For our inductive construction, let $\left\{\epsilon_{n}\right\}$ be a sequence of positive rationals tending to 0 with $\epsilon_{0}>d(X)$. Define $\mathcal{A}_{0}=\{X\}, k_{0}=1, u_{1}: \mathcal{A}_{0} \rightarrow \mathbb{Z}_{1}$ mapping $X$ to 1. 
Inductively, suppose that $\mathcal{A}_{n}$ is an invariant partition with mesh less than $\epsilon_{n}$ and for some positive integer $k_{n}$ with $n \mid k_{n}$ there is a $\mathbb{Z}_{k_{n}}$ label $u_{k_{n}}$ on $\mathcal{A}_{n}$ such that $\left[u_{k_{n}}\right]$ is a bijection from $\mathcal{A}_{n}$ to $\left[\mathbb{Z}_{1}\right] \times \mathbb{Z}_{k_{n}}$, i.e. every $f_{\mathcal{A}_{n}}$ cycle has length $k_{n}$.

Because $f \in H_{\mu}^{*}$ there exists a positive integer $k_{n+1}$ such that

$$
(n+1) k_{n} \mid k_{n+1}
$$

and an invariant partition $\mathcal{W}\left(\epsilon_{n+1}, k_{n+1}\right)$ such that every $f_{\mathcal{W}}$ cycle has length $k_{n+1}$. Choose $\tilde{u}: \mathcal{W} \rightarrow \mathbb{Z}_{k_{n+1}}$ a $\mathbb{Z}_{k_{n+1}}$ label.

Define $\mathcal{A}_{n+1}=\mathcal{W} \wedge \mathcal{A}_{n}$. So $\mathcal{A}_{n+1}$ refines $\mathcal{A}_{n}$ and since $k_{n} \mid k_{n+1}$ every $f_{\mathcal{A}_{n+1}}$ cycle has length $k_{n+1}$. We obtain a $\mathbb{Z}_{k_{n+1}}$ label, $u_{k_{n+1}}: \mathcal{A}_{n+1} \rightarrow \mathbb{Z}_{k_{n+1}}$ by composing the maps

$$
\begin{array}{r}
\left(u_{k_{n}} \circ \pi_{\mathcal{A}_{n+1}}^{\mathcal{A}_{n}}\right) \times\left(\tilde{u} \circ \pi_{\mathcal{A}_{n+1}}^{\mathcal{W}}\right): \mathcal{A}_{n+1} \rightarrow \mathbb{Z}_{k_{n}} \times \mathbb{Z}_{k_{n+1}} \\
\text { and } \quad v: \mathbb{Z}_{k_{n}} \times \mathbb{Z}_{k_{n+1}} \rightarrow \mathbb{Z}_{k_{n+1}}
\end{array}
$$

Thus, we have constructed $\mathcal{A}_{n+1} \rightarrow \mathcal{A}_{n}$ of mesh less than $\epsilon_{n+1}$ with a $\mathbb{Z}_{k_{n+1}}$ label $u_{k_{n+1}}$ such that $n+1 \mid k_{n+1}$ and $k_{n} \mid k_{n+1}$ and with $\left[u_{k_{n+1}}\right]$ a bijection. In addition, (4.73) implies that the following diagram commutes:

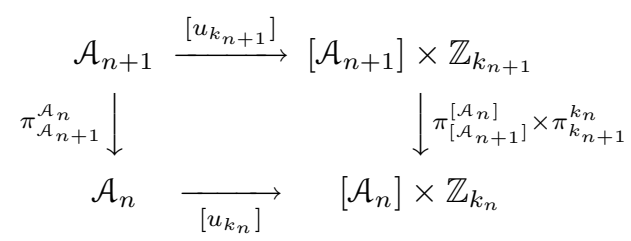

Now we identify the inverse limit spaces of these three sequences, together with their maps and measures. Since each open set in the inverse limit is a pullback of an open set from some factor, it follows that the measure on the inverse limit is uniquely determined by the projections to the factors.

For every positive integer $m$ we have $m \mid k_{n}$ if $n>m$. Hence, the adding machine group which is the inverse limit of the pointed cyclic groups $\left\{\left(\mathbb{Z}_{k_{n}}, 1\right)\right\}$ is the universal adding machine $(\Xi, g)$ and the inverse limit of the translation maps $h_{k_{n}}$ is the $g$ translation map $h$. The Haar measure $\mu_{\Xi}$ is the measure which projects to the uniform measures on the factors.

The map which associates to $x \in X$ the sequence of carriers $\pi^{\mathcal{A}_{n}}(x)$ is a surjection by compactness and it is injective because the sequence of meshes $\left\{\operatorname{mesh}\left(\mathcal{A}_{n}\right)\right\}$ tends to zero. So we obtain a homeomorphism which identifies $X$ with the inverse limit of the sequence $\left\{\mathcal{A}_{n}\right\}$. The map $f$ on $X$ projects to $f^{\mathcal{A}_{n}}$ and so $f$ is identified with the inverse limit of these maps. The measure $\mu$ is the unique measure on $X$ which projects to each $\mu_{\mathcal{A}_{n}}$.

If $\mathcal{A}$ is any partition on $X$, then it has a positive Lebesgue number $l(\mathcal{A})$. When $n$ is large enough that mesh $\left(\mathcal{A}_{n}\right)<l(\mathcal{A})$, then $\mathcal{A}_{n}$ refines $\mathcal{A}$. If $\mathcal{A}$ is fixed, then it follows that $\left[\mathcal{A}_{n}\right]$ refines $\mathcal{A}$. Thus, the sequence $\left\{\left[\mathcal{A}_{n}\right]\right\}$ is cofinal in the directed system of fixed partitions. Hence, the inverse limit of the sequence $\left\{\left[\mathcal{A}_{n}\right]\right\}$ is $X_{F}$ and the measure $\mu_{F}$ is the one with the correct projections.

From the above commutative diagram we see that on the inverse limits the sequence of bijections $\left\{\left[u_{k_{n}}\right]\right\}$ induces a homeomorphism $[u]: X \rightarrow X_{F} \times \Xi$. By (4.69) it maps $f$ on $X$ to the identity on $X_{F}$ and the translation map $h$ on $\Xi$ and the measure $\mu$ on $X$ to $\mu_{F}$ on $X_{F}$ and $\mu_{\Xi}$ on $\Xi$. 
Finally, $\mu_{F}$ is a good measure on the Cantor space $X_{F}$ and so (4.66) and Theorem 2.9 imply that there is a homeomorphism $\tilde{q}: X_{F} \rightarrow X$ which maps $\mu_{F}$ to $\mu$. The required map $q=\left(\tilde{q} \times 1_{\Xi}\right) \circ[u]: X \rightarrow X \times \Xi$.

\section{REFERENCES}

[1999] E. Akin, Measures on Cantor space, Topology Proceedings (1999) 24: 1-34. MR 2002j:28002

[2003] E. Akin, M. Hurley and J. A. Kennedy, Dynamics of topologically generic homeomorphisms, Memoirs Amer. Math. Soc \# 783 (2003).

[1988] D. Cooper and T. Pignataro, On the shape of Cantor sets, J. Diff. Geom. (1988) 28 : 203-221. MR 89k:58160

[2002] E. Glasner, Topics in topological dynamics, 1991 to 2001. Recent progress in general topology, II, 153-175, North-Holland, Amsterdam, 2002.

[1995] E. Glasner and B. Weiss, Weak orbit equivalence of minimal Cantor systems, Internat. J. Math. (1995) 6: 559-579. MR 96g:46054

[2001] E. Glasner and B. Weiss, The topological Rohlin property and topological entropy, Amer. J. of Math (2001) 123: 1055-1070. MR 2002h:37025

[1995] T. Giordano, I. F. Putnam and C. F. Skau, Topological orbit equivalence and $C^{*}$-crossed products, J. Reine Angew. Math. (1995) 469: 51-111. MR 97g:46085

Department of Mathematics, The City College (CUNy), 137 Street and Convent Avenue, New York City, New York 10031

E-mail address: ethanakin@earthlink.net 